\title{
Measuring and Modeling Execution Cost and Risk ${ }^{1}$
}

\author{
Robert Engle \\ NYU and Morgan Stanley \\ rengle@stern.nyu.edu \\ Robert Ferstenberg \\ Morgan Stanley \\ Jeffrey Russell \\ University of Chicago \\ jeffrey.russell@chicagogsb.edu
}

January 2008

\begin{abstract}
:
Financial markets are considered to be liquid if a large quantity can be traded quickly and with minimal price impact. Although the idea of a liquid market involves both a cost as well as a time component, most measures of execution costs tend to focus on only a single number reflecting average costs and do not explicitly account for the temporal dimension of liquidity. In reality, trading takes time since larger orders are often broken up into smaller transactions or when limit orders are used. Recent work shows that the time taken to transact introduces a risk component in execution costs. In this setting, the decision can be viewed as a risk/reward tradeoff faced by the investor who can solve for a mean variance utility maximizing trading strategy. We introduce an econometric method to jointly model the expected cost and the risk of the trade thereby characterizing the mean variance tradeoffs associated of different trading approaches given market and order characteristics. We apply our methodology to a novel data set and show that the risk component is a non-trivial part of the transaction decision. The conditional distribution of transaction costs is also used to construct a new measure of liquidation risk that we refer to as liquidation value at risk ( $\mathrm{LVaR})$.
\end{abstract}




\section{Introduction}

Measuring and understanding financial execution costs is important for both practitioners and regulators and has attracted substantial attention from the academic literature. Markets are considered to be liquid if a large quantity can be traded quickly and with minimal price impact. While the idea of a liquid market involves both a cost as well as a time component, most measures of execution costs tend to focus on only a single number such as bid ask spreads or realized effective spreads. A recent special issue of the Journal of Financial Markets (2003) gives a good overview of this literature including careful analysis of some of the important assumptions. See in particular, Lehmann (2003), Bessimbinder (2003), Werner (2003), Petersen and Sirri (2003).

Many strategies to reduce execution costs require taking more time to trade. This could be delaying trades to search for and take advantage of higher liquidity environments, breaking up trades to reduce spreads and price impact or using more out of the money limit orders. Recent work by Almgren and Chriss (1999, 2000) and Almgren (2003), and Grinold and Kahn (1999) point out that this added time to trade corresponds to an added risk of trading, thus introducing a risk return trade-off in execution itself. They develop families of execution strategies that lie on a risk return frontier under specific assumptions on the execution technology. More risk averse agents will trade more rapidly thus incurring higher transaction costs but lower risk. An execution strategy that takes a substantial risk should be risk adjusted before being compared with lower risk strategies. Engle and Ferstenberg (2007) embed this problem in a full portfolio optimization showing that the risk return tradeoff in portfolio theory implies a criterion for optimizing execution.

We are not aware of any paper that has shown that empirically there is a risk return trade off in order execution. While it is easy to develop trading algorithms that take more risk, it is not clear how much reduction in transaction costs, if any, can be achieved by such devices. Partly this is due to a difficulty in obtaining order data, and partly it is due to a lack of econometric techniques to simultaneously measure the first two moments of transaction costs.

In this paper we develop empirical measures of the risk and return to trading. This paper will estimate risk return frontiers for a set of trading strategies. Given estimates of the conditional risk and return of different trading strategies we can, and do, calculate risk adjusted costs of execution for investors with a particular tolerance for risk. We also examine the choice 
of trading strategy for investors with different risk tolerances and find that traders appear to select trading strategies that imply extreme risk aversion. This suggests that traders are overly risk averse in selection of their trading strategy.

This paper focuses on single orders, sometimes called parent orders, which are broken up into a sequence of smaller orders, called child orders. As the parent orders are executed, they appear in most datasets to be small transactions in a correlated order flow. However, the cost incurred by the trader is not a function of a single transaction, but rather the entire sequence of child orders. Perold (1988) introduced "implementation shortfall" as a measure of transaction costs that can accommodate such parent orders. Implementation shortfall compares the value of a paper portfolio with no transaction costs to the real portfolio obtained by actual trading. This measure of execution cost has been used in empirical work by Keim and Madhavan (1997), Bertsimas and Lo (1998), and Conrad Johnson and Wahal (2003), for example. Importantly, these studies do not consider risk as we do in our work and therefore miss an important component of liquidity. They are unable to risk adjust the transaction costs.

Our data consists of a sample of 233,913 orders executed by Morgan Stanley in 2004. We use this data to construct measures of both execution risk and cost ${ }^{2}$ conditional on order and market characteristics.

The paper is organized as follows. Section 2 discusses the choice of trading strategy by the investor. Section 3 introduces measures of order execution cost and risk. Section 4 presents the data used in our analysis and some preliminary analysis. Section 5 presents a model for conditional cost and risk along with estimates while section 6 employs diagnostic tests to develop a richer specification. Section 7 explicitly examines the economic importance of transaction risk, section 8 presents an application of the model to liquidity risk and section 9 concludes.

\section{Choice of trading strategy}

Investors are offered a wide choice of strategies for executing a particular order. The order can be executed by a block trader from one of many brokerage houses or by algorithmic trading from many different vendors. Within each of these categories there are a range of options which might entail some risk sharing with the broker, some specific instructions, some specific venue, a particular crossing strategy, or many other considerations. Each of these possibilities could give an estimate of the expected cost and the expected risk of the trade. The investor would then use

\footnotetext{
2 The data does not include the identities of the traders.
} 
an appropriate utility function to determine the best way to execute the trade. It could turn out that one of the options is not to execute the order at all and this could also be an outcome.

In order to do this optimization, each venue must be associated with a risk and return estimate for all types of orders. That is, given the name, quantity, day and instructions, what is the outcome expected to be? Not all venues provide such information. Because this is a repeating business, however, it is possible for clients to construct their own estimates. This can also be useful because it gives a way to evaluate the success of brokers in achieving the performance they advertise.

The building blocks for this decision analysis are the expected cost and risk as a function of a set of characteristics specific to each venue. It is possible that there will be some characteristics of an order that are not observable to the econometrician or possibly to the broker. Some orders may be based on information on upcoming announcements or other forthcoming trades, possibly from the same investor. These orders might have performance that deviates from the average in either mean or risk.

If there are important unobserved characteristics of orders, then the function predicting the mean and variance of transaction cost will inappropriately attribute these trades to correlated observable characteristics. In particular, these unobservable characteristics could be associated with trading instructions. For example, if an announcement is expected very soon, then the order could be called urgent and would be traded fast with less concern for cost. Consequently, orders labeled urgent may on average have worse execution in either mean or variance than would be expected given the trading environment. The situation is like errors in variables or simultaneous equations bias. Although there will be a bias in the coefficients from the structural relation, the estimated coefficients will still give an unbiased estimate of the conditional expectation, and perhaps that is the most useful result. This endogeneity has always been present in trade data and we have hundreds of studies of trade execution that appear to be reliable and reproducible. Many investors, who issue urgent instructions because they believe they have time sensitive information, may have less information than they think and consequently are simply paying too much for their executions. There is evidence in our data set for this position. As in simultaneous equation estimation, a small endogeneity leads to a small bias. In this paper we will assume that the unmeasured characteristics of trades are relatively small and uncorrelated with the observable characteristics, and we interpret the results as the expected costs and risks of a typical trade with particular characteristics when executed using a certain strategy. 


\section{Measuring order execution cost and risk.}

This section defines the transaction cost measure and shows how it relates to the portfolio risk and return. Let the position measured in shares at time period $t$ be $x_{t}$. The number of shares transacted in period $t$ is simply the change in $x_{t}$ so that purchases are positive and sales are negative. Let $p_{0}$ denote the fair market value of the asset at the time of the order arrival; in practice this will be the midquote at the time of the order arrival. Let $\tilde{p}_{t}$ denote the transaction price of the asset in period $t$ which will in general depend upon the size, direction and timing of the trades. Trading takes place over the time interval $[0, \mathrm{~T}]$ where $x_{0}$ denotes the initial position prior to trade and $x_{T}$ the position post trade. The transaction cost for a given parent order is then defined to be:

$$
T C=\sum_{t=1}^{T} \Delta x_{t}\left(\tilde{p}_{t}-p_{0}\right)
$$

Notice that this is simply the cost of acquiring $\left(x_{T}-x_{0}\right)$ shares with actual transaction prices minus the cost of acquiring these shares at the initial fair market price of $p_{0}$. Transactions that occur above the reference price will therefore contribute positively toward transaction costs when purchasing shares. Alternatively, when liquidating shares, the change in shares will be nonpositive, hence transaction prices that occur below the reference price will contribute positively to transaction costs. For a given order, the transaction costs can be either negative or positive depending upon whether the price moved with or against the direction of the order. However, because each trade has a price impact that tends to move the price up for buys and down for sells, we would expect the parent transaction cost to be positive on average. This transaction cost is the realization of a random variable with both a mean and variance that can be estimated. A measure of the transaction cost per dollar traded is obtained by dividing the transaction cost by the arrival value:

$$
T C \%=\frac{T C}{\left|x_{T}-x_{0}\right| p_{0}}
$$

This measure allows for more meaningful comparison of execution cost for different stocks with different prices and therefore serves as the basis of our empirical work.

Engle and Ferstenberg (2007) further show that the change in portfolio value, $y_{t}$, over this period can be expressed as

$$
y_{T}-y_{0}=x_{T}{ }^{\prime}\left(p_{T}-p_{0}\right)-T C
$$


where $x_{t}$ and $p_{t}$ are now vectors with $\mathrm{j}^{\text {th }}$ element denoting the quantity and price respectively of asset $j$ held at time t. TC is the multi-asset generalization of (1). The return on the portfolio is simply the capital gain if the trade had been executed immediately with no cost, minus the transactions cost as defined above. In several important cases, this gives direct implications for execution evaluation. When this trade is a liquidation, the vector $x_{T}$ has all zero elements and only $T C$ is relevant. This could be a liquidation of a short or a long position so buys or sells could be analyzed. The tolerance for risk during execution is thus exactly the same as the tolerance for risk in portfolio selection. This situation could correspond to an institutional investor who takes on the risk of executing block trades for clients and is always moving toward a cash position. As discussed later, this could also correspond to the risk management of a portfolio which is evaluated by its liquidation value rather than by a mark to market value.

When a portfolio includes assets that are not being traded and when the final portfolio holds shares, then the variance of the portfolio includes more than just the variance of transactions costs. There is generally a covariance between portfolio returns and transaction cost that should be considered in designing optimal executions. Engle and Ferstenberg show that in the important case where $x_{T}$ is chosen by traditional mean variance optimization, there are no covariances so that the relevant risk is simply the variance of TC. Hence in the leading cases, a mean variance trader should optimally trade off the mean and variance of $T C$ when deciding how to transact.

IV. The data

Analysis of the execution cost of an order requires detailed order execution data including the arrival price, the parent trade size and the volume weighted average of the transaction prices that were used to fill a given order. Such data was supplied by Morgan Stanley. We do not know the identity of the traders that placed these orders. The orders could have been initiated by Morgan Stanley traders on behalf of their clients or by a buy side trader on behalf of a portfolio manager. We use the word "trader" to refer to either one.

The orders were executed by Morgan Stanley’s Benchmark Execution Strategies ${ }^{\mathrm{TM}}$ (BXS) strategies during 2004. We consider two types of orders. The first is the arrival price (AP) strategy that minimizes the expected cost of the trade (TC\%) for a given level of risk where the midquote are taken as the benchmark efficient price. The algorithm attempts to choose a minimal cost trading trajectory that consists of a sequence of trade sizes times subject to the risk constraint. Trades are then executed using an automated trading procedure that is a variant of 
Almgren and Chriss (2000). The second is a volume weighted average price strategy. This strategy attempts to trade as close as possible to the volume weighted average price over a specified time interval.

For the AP strategy, the trader chooses one of three urgency levels - high, medium, and low. The level of urgency is inversely related to the level of risk that the trader is willing to tolerate. High urgency orders have relatively low risk, but execute at a higher average cost obtained by trading very quickly. The medium and low urgency trades execute with progressively higher risk but at a lower average cost obtained by trading more slowly, over a longer period of time. The trader chooses the urgency and the algorithm derives the time to complete the trade given the state of the market and the trader's constraints. Given order size and market conditions, lower urgency orders tend to take longer to complete than higher urgency trades. However, since the duration to completion depends upon the market conditions and other factors, there is not perfect correspondence between the urgency level and the time to complete the order. All orders in our sample, regardless of urgency, are filled within a single day.

For VWAP strategies, the trader selects a time horizon and the algorithm attempts to execute the entire order by trading proportional to the market volume over this time interval. We only consider VWAP orders where the trader directed that the order be filled over the course of the entire trading day or that the overall volume traded was a very small fraction of the market volume over that period. This can be interpreted as a strategy to minimize cost regardless of risk. As such, we consider this a risk neutral trading VWAP strategy. This need not correspond to a risk neutral strategy, but clearly the intent of the trader is consistent with risk neutral VWAP trading. Generally, these orders take longer to fill than the low urgency orders and should provide the highest risk and the lowest cost.

We consider orders for both NYSE and Nasdaq stocks. Several filters are applied to our transaction data. We only consider completed orders. Hence orders that begin to execute and are then cancelled midstream are not included in order to ensure homogeneity of orders of a given type. We excluded short sales because the uptick rule prevents the economic model from being used "freely". We do not consider orders executed prior to 9:36 since the market conditions surrounding the open are quite different than non-opening conditions. Only stocks that have an arrival price greater than $\$ 5$ are included - we find that very cheap stocks have transaction prices that are impacted by price discreteness and may require special modeling techniques appropriate for such data. Orders that execute in less than 5 minutes tend to be very small orders that may be traded in a single trade and are not representative of the mean variance tradeoff that is of interest in our work. For similar reasons, orders smaller than 1000 shares are also not included. Finally, 
orders that are constrained to execute more quickly than the algorithm would dictate due to the approaching end of the trading day are also excluded. In the end, we are left with 233,913 parent orders.

For each order we construct the following statistics. The percent transaction costs are constructed using equation (2). We further construct the 5 day lagged bid-ask spread weighted by time as a percent of the midquote, the annualized 21 day lagged close to close volatility and the order shares divided by the lagged 21 day median daily volume. Table 1 presents summary statistics of our data. The statistics weight each order by its fraction of dollar volume.

The rows labeled B and S break down the orders into buyer and seller orders respectively. $62 \%$ of the dollars traded were buys and $38 \%$ sells. Buy orders tend to be slightly more expensive on average in this sample. The risk is similar. We see that $75 \%$ of the dollar volume was for NYSE stocks and 25\% for Nasdaq. We see that NYSE orders tend to cost less than Nasdaq by an average of about 5 basis points. It is important to note that these statistics are unconditional and do not control for differences in characteristics of the stocks traded on the two exchanges which might be driving some of the variation in the observed costs. For example, we see that the average volatility of Nasdaq stocks is substantially higher than that of NYSE.

The last four rows separate the orders by urgency. H, M, and L, correspond to high, medium and low urgencies and V is the VWAP strategy. Hence as we move down the rows we move from high cost, low risk strategies to low cost, high risk strategies. Almost half of the orders are the risk neutral VWAP strategy (46\%). Only 10\% of the order volume is high urgency, $24 \%$ is medium urgency and $20 \%$ is high urgency. This is reflected in the sample statistics. The average cost decreases from 11.69 basis points to 8.99 basis points as we move from high to low urgency orders. At the same time, the risk moves from 12.19 basis points up to 40.89 basis points for the same change in urgency. Contrary to the intent, the VWAP strategy does not exhibit the lowest cost at 9.69 basis points. It is the most risky however. Of course, the order submission may depend on the state of the market and characteristics of the order. These unconditional statistics will not reflect the market state and may blur the tradeoffs faced by the traders.

Table 2 presents the same summary statistics conditional on the size of the order relative to the 21 day median daily volume. The first bin is for orders less than a quarter of a percent of the 21 day median daily volume and the largest bin considered is for orders that exceed $1 \%$. For each bin the statistics are presented for each type of order. The top of the table is for NYSE and the bottom half of the table is for Nasdaq stocks. Not surprisingly, for each order type, larger order sizes tend to be associated with higher average cost and higher risk indicating that larger orders are more difficult to execute along both the cost and risk dimensions. 
This tradeoff can be seen clearly by plotting the cost/risk tradeoffs for each of the percent order size bins. Figure 1 presents the average cost/risk tradeoff for the NYSE stocks. Each contour indicates the expected cost/risk tradeoff faced for a given order size. Each contour is constructed using 4 points, the three urgencies and the VWAP. For a given contour, as we move from left to right we move from the high urgency orders to the VWAP. Generally speaking, the expected cost falls as the risk increases. This is not true for every contour, however. Increasing the percent order size shifts the entire frontier toward the north east indicating a less favorable average cost / risk tradeoff. Figure 2 presents the same plot but for Nasdaq stocks.

Contrary to what might be expected, some order size bins exhibit a cost increase as we move to less urgent strategies. These plots, however, do not consider the state of the market at the time the order is executed. It is entirely possible that the traders consider the state of the market when considering what type of urgency to associate with their order. If this is the case, a more accurate picture of the tradeoff faced by the trader can be obtained by considering the conditional frontier. This requires building a model for the expected cost and the standard deviation of the cost conditional on the state of the market and is the goal of the next section of the paper.

V. Modeling the expected cost and risk of order execution.

Both the transaction cost and risk associated with executing a given order will vary according to characteristics of the order and the state of the market. In this section we propose a modeling strategy for both the expected cost and risk of trading an order. In this way, we build a model for the conditional mean variance tradeoff obtained by each trading strategy. The model is estimated using the Morgan Stanley execution data described in the previous section.

We now propose a joint model for the conditional mean and variance of transaction costs. Both the mean and the variance of transaction costs are assumed to be an exponential function of the market variables and the order size. Specifically the transaction costs for the $i^{\text {th }}$ order are given by:

$$
T C \%_{i}=\exp \left(X_{i} \beta\right)+\exp \left(\frac{1}{2} X_{i} \gamma\right) \varepsilon_{i}
$$

where $\varepsilon_{i} \sim$ iid $N(0,1)$. The conditional mean is an exponential function of a linear combination of the $X_{i}$ with parameter vector $\beta$. The conditional standard deviation is also an exponential function of a linear combination of the $X_{i}$ with parameter vector $\gamma . X_{i}$ is a vector of conditioning 
information. In our empirical work we find that the same vector $X_{i}$ explains both the mean and the variance but this restriction is obviously not required.

Clearly the variance must be a positive number and while the realization of transaction costs can be either positive or negative (and empirically we do find both signs), the expected transaction cost should be positive. The exponential specification for both the mean and the variance restricts both to be positive numbers.

We consider several factors that market microstructure theory predicts should contribute to the ease/cost of executing a given order. The lagged 5 day time weighted average spread as a percent of the midquote. The daily volatility constructed from the average close to close returns over the last 21 days. The average historical 21 day median daily dollar volumes. In addition to these market variables we also condition on the log of the dollar value of the order and the urgency associated with the order. The urgency is captured by 3 dummy variables for high, medium, and low urgencies. The constant term in the mean and variance models therefore corresponds to the VWAP strategy.

The exponential specification for the mean with an additive disturbance, is not a model we have seen previously used in the literature. The often used method of modeling the logarithm of the left hand side variable won't work here because the transaction cost often takes negative values. Despite the exponential form for the mean and variance, interpretation of the parameters is straight forward. Notice that $\ln \left[E\left(T C \%_{i}\right)\right]=X_{i} \beta$. Hence the coefficients can be interpreted as the percent change in expected TC\% for a one unit change in X. Coefficients of right hand side variables that are expressed as the logarithm of a variable (such as $\ln ($ value)) can be interpreted as the elasticity with respect to the non-logged variable (such as value).

The exponential model also allows for interesting nonlinear interactions that we might suspect should be present. Consider the expected transaction cost and the logged value (dollar size of the trade) and volatility variables. We have $E(T C \%)=\exp ^{(\text {other variables })} v$ alue $e^{\beta_{1}}$ volatility $^{\beta_{2}}$. If $\beta_{1}$ is larger than 1 then the cost increases more than proportionally to the value. If $\beta_{1}$ is smaller than 1 then the expected cost increases less than proportionally to the value. If $\beta_{1}$ and $\beta_{2}$ are both positive, then increases in the volatility result in larger increases in the expected cost for larger value trades. Alternatively, as the value of the trade goes to zero, so does the expected cost which seems reasonable. It is entirely possible that the marginal impact of volatility might be different for different order sizes. The exponential model allows for this possibility in a very parsimonious fashion. Hence, what appears as a very 
simple nonlinear transformation allows for fairly rich nonlinear interactions. Obviously, using the exponential function for the variance has the same interpretation.

We estimate the model by maximum likelihood under the normality assumption for $\varepsilon$. It is well known that under standard regularity conditions the normality assumption delivers a quasi maximum likelihood estimator. As long as the conditional mean and variance are correctly specified, we still obtain consistent estimates of the parameters even if the normality assumption is not correct. The standard errors, however, will not be correct in the event that the errors are not normal. Robust standard errors that are consistent in the event of non-normal errors can be constructed following White (1982) and are constructed for our parameter estimates. The information matrix is block diagonal between the parameters of the mean and the parameters of the variance, hence the estimated parameters for the mean and variance equations are asymptotically independent.

\section{Empirical Results}

The estimation is performed separately for NYSE and Nasdaq stocks. The two markets operate in a very different fashion and it is unlikely a single model would be appropriate for both trading venues. We have 166,508 NYSE orders and 67,405 Nasdaq orders. We do not separate VWAP and AP orders, however we will use the order type in the conditioning information set. We find that empirically this seems reasonable.

We begin by estimating a simple, parsimonious model that allows for particularly easy interpretation and comparison across NYSE and Nasdaq models. The model uses the same explanatory variables for both the mean and the variance. Although this simple model actually performs quite well, we later consider more elaborate models that can extract richer information about differences between Nasdaq and NYSE order execution.

The simple model contains the spread, the logarithmic daily volatility, the logarithmic volume, indicator variables for the urgency of the trade and a VWAP indicator variable. Estimation results for the variance and mean equations for NYSE transactions are given in given in tables 3 and 4 respectively. Tables 5 and 6 present the model estimates for Nasdaq transactions.

The estimated coefficients are remarkably similar for the NYSE and Nasdaq models. The spread coefficient is positive in both the mean and variance equations. Since higher spreads tend to be associated with less liquid markets it is not surprising to find that wider spreads are associated with both higher risk and cost - more difficult orders. It is also interesting to see that 
the coefficient on the spread in the expected cost equations is nearly 1 for both NYSE and Nasdaq orders. This indicates that, all else equal, if the spread doubles, so does the expected cost - not a surprising result. Higher volatility stocks are more costly to transact in and, not surprisingly, have higher risk. In fact, we see that the coefficient on the log volatility is nearly one. Recall that the model is specified in terms of the transaction cost variance, not volatility (standard deviation). This implies that the coefficient on the traded assets log variance is half the coefficient on its log volatility.

If the parent order is traded over a fixed time period by trading equal amounts in each time interval, then we would expect the variance of transaction cost would be proportional to the variance of the asset variance. This strategy however does not attempt to minimize risk. A more sophisticated strategy will accelerate trades when the volatility is high thereby shortening transaction times. Thus we are not surprised to find that transaction cost variance increases less than proportional with asset variance.

Not surprisingly, the expected transaction costs are an increasing function of the volatility. In order to maintain a given level of trading risk, orders for higher volatility stocks must be executed more quickly than low volatility stocks. This shorter execution time induces higher expected transaction costs.

The value and volume coefficients are roughly of equal magnitude but opposite sign in both the expected cost and variance equations for both NYSE and Nasdaq. This is not surprising since the size (value) of the order must be considered relative to the typical daily volume of the traded asset. The magnitudes and signs of the estimated coefficient imply that it is the ratio of the order size to the daily volume that is useful in predicting both cost and risk. The estimated coefficients are all around .5 in magnitude indicating that both the risk and cost increase roughly in the square root of the order size expressed as a fraction of daily volume.

Figures 3 and 4 present the expected cost and the standard deviation (measured in basis points) of transaction costs as a function of the order size relative to the daily volume. Normalizing by volume allows us to compare order sizes across stocks with different typical daily volumes in a more meaningful way. The plots consider order sizes ranging from near 0 percent up to $2 \%$ of the daily volume and represent an average stock on an average day. Figures 5 and 6 present the same plots, but for the Nasdaq stocks. In the expected cost plots, the higher curves correspond to the more urgent orders. The opposite is true for the standard deviation plots. The model estimates indicate that both the expected cost and risk are concave functions of the order size expressed as a fraction of the daily volume. Hence order size increases both expected cost and variance but at a decreasing rate. 
The urgency dummy variables appear as expected. We see that the expected cost increases as the urgency level increases and that the risk decreases with higher urgency orders. These parameters can be more easily interpreted in the context of the mean variance plots previously used for the unconditional moments. We again consider the risk/cost tradeoff for an average stock under average conditions for NYSE and Nasdaq markets. These contours are plotted in figures 7 and 8 . The ellipses represent 95\% confidence intervals for the true mean and true variance for each order submission strategy. As we move from left to right we move from high urgency to medium, to low and finally VWAP or the risk neutral strategy. The plots suggest that moving from low urgency to the VWAP strategy tends to increase risk with little reduction in cost both for NYSE and Nasdaq stocks. We show later in the paper that the VWAP strategy might be preferred after risk adjusting cost.

We can evaluate the cost/risk tradeoff for the characteristics of any stock and any order characteristic. To get an idea of how this tradeoff varies as the order size relative to daily volume varies we next plot the mean variance frontiers for different value ratios. We again consider a typical stock on a typical day. These plots are presented in figures 9 and 10 for typical NYSE and Nasdaq stocks respectively. The larger orders shift the cost/risk tradeoff to less desirable north east region. We again see that the order size effects on the cost/risk tradeoff are substantial.

It is tempting at this point to compare the mean variance frontiers obtained for orders filled on Nasdaq and orders filled on the NYSE. In doing so, we can compare market quality using a measurement that reflects real world trading and both expected cost and risk components of execution cost. We use the estimated models to compare the mean variance frontiers for the same transaction under identical market conditions on the NYSE and on the Nasdaq. Most market and order characteristics included in the model are comparable across the two markets (i.e. volatility is volatility, spreads are spreads). The volume however is not easily comparable across the two markets. The problem is that much of the volume recorded for Nasdaq trades is not volume that Morgan Stanley could have participated in. Some trades are directly facilitated between two dealers in order to rebalance inventories and some of the volume on Nasdaq took place on satellite markets not available to Morgan Stanley. This is a well known problem and some estimates put Nasdaq volume at about 30\% overstated relative to NYSE. Figure 11 presents the comparison with no adjustments to the volume. Figure 12 presents the comparison with the Nasdaq volume 30\% greater than the NYSE volume. This adjustment would be the correct if volume on Nasdaq were in fact $30 \%$ overstated as conjectured. Although the NYSE appears to have lower cost for a given risk level, across all risk levels we see that once the volume 
differences are accounted for there is little difference between the market qualities implied by our models.

Finally, despite the similarity in the Nasdaq and NYSE models, we find that the models are statistically different. We test the null hypothesis that both the mean and variance models are identical for the NYSE and Nasdaq orders. This null hypothesis is tested by a likelihood ratio test based on the difference between the sum of the likelihoods for the two unrestricted NYSE and Nasdaq models and the restricted model using the pooled data. Twice the difference in these two likelihoods will have a chi-squared distribution with degrees of freedom given by the number of restricted parameters, or 16. Twice the difference in the two likelihoods is 1954.76. The critical value is 26.29 so we overwhelmingly reject the null with a p-value near 0 . Hence, while the models are qualitatively similar, there are statistically meaningful quantitative differences.

\section{Specification Tests}

This model appears to do a nice job of relating the expected cost and risk components to the characteristics of the stock traded and the market as well as characteristics of the order. The parameters are easily interpreted and comparable across models. Nevertheless, specification tests suggest that more elaborate models could be considered. While the interpretation of the models may become slightly more complex, more elaborate models might highlight differences between the NYSE and Nasdaq stocks and more details of how market and order characteristics impact execution cost and risk.

We considered two extensions of the model, additional nonlinear terms and interactions between variables. The additional non linear terms considered are the value, volume, volatility variables in levels (ie not logarithmic) and the logarithmic spread. A natural extension to consider is that the VWAP orders have different properties than the AP orders. This can be tested by interacting the VWAP dummy with the variables already considered in the more parsimonious model presented earlier. This model is the most general we consider and it has many more variables in both the mean and the variance.

We proceeded to calculate Lagrange Multiplier tests for omitted variables of this general model. The null model is tested against the general model and if it is rejected, additional variables are added to the base model until the test fails to reject. In most cases, the variables with the highest t-statistics in the test are added first.

The LM tests are performed separately on the mean and variance equation for each market and are easily computed. Specifically, for each observation in the sample, the 
standardized residual $\hat{\varepsilon}_{i}$ is given by $\hat{\varepsilon}_{i}=\frac{T C \%_{i}-\hat{\mu}_{i}}{\hat{\sigma}_{i}}$ where $\hat{\mu}=\exp \left(X_{i} \hat{\beta}\right)$ and $\hat{\sigma}_{i}=\exp \left(\frac{1}{2} X_{i} \hat{\gamma}\right)$ and $X_{i}$ is a vector of variables included in the null model. A test for an omitted variable in the mean equation is obtained from $\mathrm{T}^{*} \mathrm{R}$-squared obtained from the least squares regression

$$
\hat{\varepsilon}_{i}=\frac{\hat{\mu}_{i}}{\hat{\sigma}_{i}} X_{i} \theta_{0}+\frac{\hat{\mu}_{i}}{\hat{\sigma}_{i}} Z_{i} \theta_{1}
$$

where $Z_{i}$ is a vector of candidate omitted variables. The test examines whether the standardized residual is correlated with any candidate missing variables using the heteroskedasticity correction estimated under the null. Each candidate variable appears as the derivative with respect to the coefficient divided by the estimated heteroskedasticity. The test statistic has the usual Chisquared distribution with degrees of freedom given by the number of candidate variables in $Z_{i}$. The test for omitted terms from the variance model is similarly obtained by the least squares regression of.

$$
\hat{\varepsilon}_{i}^{2}=X_{i} \phi_{0}+Z_{i} \phi_{1}
$$

Again, the intuition here is that the test looks for any correlation between the squared standardized residual and potentially omitted variables in $Z_{i}$. This expression is particularly simple as the variance terms cancel. All tests are performed at the $5 \%$ level.

Tables 7 and 8 present the parameter estimates for the final, more detailed model that passes the LM tests against the general alternative. The final model for the variance contains the same variables for the NYSE orders and the Nasdaq orders although there are some differences in the values of the coefficients. The models for the expected cost are different.

We begin our discussion with the variance models. The additional "nonlinear" terms are the value, volatility, volume, (in levels) and the log spread. The elasticity of transaction risk with respect to the size of the order is increasing in the order size. The volatility and volume variables offset the logged terms so that larger volatility and volume result in elasticities that are smaller (in absolute value).

There are three VWAP interaction terms with log volatility, log volume, and log value. The signs on the log value and log volume are opposite the sign of the non-interacted counterparts. This means that VWAP orders are less sensitive to the order size (as a fraction of daily volume) than non VWAP orders. This makes sense since the VWAP orders do not directly take risk into account but rather complete the trade over a specified time interval. The VWAP 
term interacted with the log volatility is negative for the Nasdaq orders, but positive and marginally significant for the NYSE orders. The volatility impact for both models is very small relative to the non-interacted variable.

More pronounced differences between NYSE and Nasdaq occur in the models for the expected cost. The log of the spread is needed in the expected cost equation for Nasdaq, but not for NYSE. Nasdaq spreads are more informative than NYSE spreads regarding transaction costs since most transactions in Nasdaq occur at the bid or the ask, unlike the NYSE where many transaction prices occur strictly inside the spread. Perhaps it is not surprising then that the Nasdaq model has a more complex dependence on the spread. The coefficient is positive on the log spread variable indicating that expected costs should be more sensitive, and decrease more quickly for the smallest spread stocks than found for NYSE stocks. The log volume term is interacted with the VWAP. This suggests that VWAP trades are more sensitive to volume and receive greater benefit than non-VWAP trades. Again, this could be due to the fact that VWAP trades do not optimize with respect to trading costs or risk. The benefits of high volume are therefore more important here to overcome the lack of optimization inherent in the strategy. The other additional variables, while statistically significant are small in terms of economic impact.

VIII. Is the risk component economically meaningful?

In this section we examine the economic implications of the risk component. We ask two questions. First, how important is the transaction risk component in the transaction decision? Second, how what kind of risk aversion is consistent with traders execution choices?

In order to address these questions we consider a trader with expected utility given by $E(U)=-E(T C)-\lambda V(T C)$. This is the standard mean variance utility but since TC denotes a transaction cost and not a return we take the negative. We ask how much money would the trader be willing to pay in order to transact risklessly. In this setting, $\lambda V(T C)$ represents the amount of expected costs the trader would be willing to incur in order to transact risklessly. Similarly, the risk adjusted transaction cost can be defined as

$$
T C^{R A}=T C-\lambda V(T C)
$$

Obviously this depends upon the choice of the risk aversion parameter $\lambda$. There is a large literature that argues that this parameter may range from 0 to 15 . See for example Friend and Blume (1975) and Chou, Engle and Kane (1992) for empirical estimates. A reasonable value is 3 although this may suggest substantial risk tolerance. Considerably higher values should also be 
considered. Figure 13 presents a plot of the cost of risk for NYSE and Nasdaq stocks for the four different trade types. The VWAP trades have the highest cost of risk at about half a basis point and the high urgency trades have the smallest cost of risk at about $1 / 20^{\text {th }}$ of a basis point. A typical trade has an expected cost of around 4 basis points. For higher urgency trades the cost of risk is non-trivial. Furthermore, the small effects will be compounded when a portfolio is actively managed.

We next examine what the choice of execution strategy implies about the traders risk aversion. Since the frontier is piecewise linear, a range of values of $\lambda$ will be consistent with a given strategy yielding optimal utility. We solve for the range of values of $\lambda$ for a typical trade under normal conditions. We also consider large orders $\left(10^{\text {th }}\right.$ percentile) and small orders $\left(90^{\text {th }}\right.$ percentile). The results are given in table 9. We find that VWAP is consistent with realistic values of $\lambda$, typically smaller than 3 . The low urgency trades are also often consistent with reasonable risk aversion levels, but the medium and high urgency trades are not, generally requiring exceptionally high risk aversion to be optimal. Interestingly, we find that traders often use strategies that imply extreme risk aversion. The Nasdaq conditional frontier is not completely convex so that medium urgency orders are not optimal for any value of $\lambda$.

\section{The Distribution of Transaction Cost and Liquidation Value at Risk (LVaR)}

Liquidation risk is the uncertainty about how much it costs to liquidate a position in a timely manner. Liquidation risk is important from both an asset management/risk perspective, as well as a more recent literature on asset pricing and liquidity (see for example Easley and O’Hara (2003), Pastor and Stambaugh (2003), Pedersen and Acharya (2005)). The conditional distribution of transaction costs provides a detailed description of liquidation risk. For a given position, liquidation execution strategy, and set of market conditions our model describes the probability distribution of the cash value of the position after liquidating. One measure that summarizes the liquidation risk is the liquidation value at risk or $\mathrm{LVaR}$. Like the traditional value at risk (VaR), LVaR tells us the minimum number of dollars that will be lost with some probability $\alpha$, when liquidating an asset.

For a given liquidation order the conditional mean and variance can be constructed. Under a normality assumption, one can construct an $\alpha \%$ LVAR given by:

$$
\operatorname{LaR}(\alpha)=\exp (X \hat{\beta})+\exp \left(\frac{1}{2} X \hat{\gamma}\right) z_{1-\alpha}
$$


where $z_{1-\alpha}$ is the $1-\alpha \%$ quantile.

More generally, we might not wish to impose the normality assumption and instead use a semi-parametric approach. In the first stage, consistent estimates or the parameters can be estimated by QMLE. In the second stage, the standardized residuals can be used to construct a non-parametric estimate of the density function of the errors $\varepsilon$. The standardized residuals are given by:

$$
\hat{\varepsilon}_{i}=\frac{T C \%_{i}-\exp \left(X_{i} \hat{\beta}\right)}{\exp \left(\frac{1}{2} X_{i} \hat{\gamma}\right)}
$$

A non-parametric estimate of the density or perhaps just the quantiles themselves can then be used to construct a semi-parametric LVaR. Specifically, let $\hat{\varepsilon}_{1-\alpha}$ denote a non-parametric estimate of the $\alpha \%$ quantile of the density function of the error term $\varepsilon$. Then the semi-parametric $\alpha \%$ LVAR is obtained by replacing $z_{1-\alpha}$ with the non-parametric quantile $\hat{\varepsilon}_{1-\alpha}$ :

$$
\operatorname{LaR}(\alpha)=\exp (X \hat{\beta})+\exp \left(\frac{1}{2} X \hat{\gamma}\right) \hat{\varepsilon}_{\alpha}
$$

Figures 14 and 15 present the standardized residuals for the NYSE and Nasdaq models. The residuals are clearly non-normal. We use the empirical quantiles of the data to construct the LVaR. Figures 16 and 17 present the $1 \%$ LVaR associated with the high, medium and low urgency orders as well as the VWAP. The LVaR estimates are constructed for typical stocks on a typical day. The vertical axis is the transaction cost in basis points. As we move from left to right we move from LVaR to low urgency to the high urgency orders. The LVaR is given by the upper bar for each order type. The expected cost for each order type is given by the smaller bar in near the origin. The differences in the mean are small relative to the changes in the risk across the different order types. Since the risk dominates, the minimum LVaR order type here is given by the most aggressive strategy, the high urgency order. The $1 \% \mathrm{LVaR}$ for this order type is just under around half a percent for NYSE and 1\% for Nasdaq. For each order type the lower dashed line completes a $98 \%$ prediction interval.

\section{Conclusion}

This paper demonstrates that expected cost and risk components of transaction costs can be estimated from detailed transaction data. We show how to build mean variance frontiers for different trading strategies. We find that the (conditional) expected cost and risk components can 
be modeled using an exponential specification for the mean and variance. Characteristics of the order and state of the market play an important role in determining the cost/risk tradeoff faced by the trader.

After controlling for order and market characteristics we show that for moderate utility functions the value of the risk is considerably smaller than the value of the mean of transaction cost, but is non-negligible and can compound when portfolios are actively managed. In an important step forward in the analysis of execution costs, we show how to risk adjust execution costs and find that the traders often appear very risk averse when choosing an execution strategy.

Our model for the conditional distribution of transaction costs completely describes liquidation risk. We provide an example of how this conditional distribution can be used to assess portfolio liquidation risk using the notion of liquidation value at risk (LVAR). There are, of course, many other possible measures of liquidation risk that could be constructed using the conditional distribution.

This general approach can be used to compare various execution technologies, exchanges and liquidity search engines and it should prove a useful tool for clients to compare the performance of alternative brokers or algorithmic traders. Unlike existing measures, the proposed approach accounts for both average cost and, importantly, risk. When embedded in a portfolio model it allows stock selection with better control of execution costs. 


\begin{tabular}{|c|c|c|c|c|c|c|c|c|c|c|c|c|c|c|c|}
\hline Exchange & Side & Benchmark & Urgency & Weight & Count & Price & Spread & Volatility & Volume & & $\begin{array}{l}\text { pitalization } \\
(000)\end{array}$ & $\begin{array}{l}\text { Order } \\
\text { Value }\end{array}$ & $\begin{array}{l}\text { Order } \\
\text { Shares }\end{array}$ & $\begin{array}{l}\text { Cost } \\
\text { (BP) }\end{array}$ & $\begin{array}{c}\text { StDev } \\
\text { (BP) }\end{array}$ \\
\hline & & & & $100 \%$ & 233,913 & $\$ 45.07$ & $0.09 \%$ & $26 \%$ & $1.59 \%$ & $\$$ & $59,609,060$ & $\$ 310,472$ & 9,154 & 10.09 & 47.24 \\
\hline & B & & & $62 \%$ & 147,649 & $\$ 45.06$ & $0.09 \%$ & $26 \%$ & $1.57 \%$ & $\$$ & $58,137,900$ & $\$ 302,812$ & 8,946 & 10.77 & 47.17 \\
\hline & $S$ & & & $38 \%$ & 86,264 & $\$ 45.09$ & $0.08 \%$ & $26 \%$ & $1.62 \%$ & $\$$ & $61,965,453$ & $\$ 323,583$ & 9,512 & 8.99 & 47.31 \\
\hline NYSE & & & & $75 \%$ & 166,508 & $\$ 48.01$ & $0.09 \%$ & $23 \%$ & $1.68 \%$ & $\$$ & $66,717,110$ & $\$ 326,031$ & 8,701 & 8.82 & 43.28 \\
\hline NASDAQ & & & & $25 \%$ & 67,405 & $\$ 36.38$ & $0.08 \%$ & $36 \%$ & $1.33 \%$ & $\$$ & $38,565,201$ & $\$ 272,037$ & 10,273 & 13.84 & 57.19 \\
\hline & & A & $\mathrm{H}$ & $10 \%$ & 15,616 & $\$ 47.81$ & $0.08 \%$ & $26 \%$ & $1.18 \%$ & $\$$ & $60,838,460$ & $\$ 475,462$ & 12,845 & 11.69 & 23.19 \\
\hline & & A & M & $24 \%$ & 54,095 & $\$ 44.73$ & $0.09 \%$ & $27 \%$ & $1.47 \%$ & $\$$ & $48,482,781$ & $\$ 320,909$ & 9,688 & 11.09 & 32.20 \\
\hline & & A & $\mathrm{L}$ & $20 \%$ & 51,588 & $\$ 46.44$ & $0.08 \%$ & $26 \%$ & $1.13 \%$ & $\$$ & $68,894,693$ & $\$ 285,018$ & 8,106 & 8.99 & 40.89 \\
\hline & & V & & $46 \%$ & 112,614 & $\$ 44.04$ & $0.09 \%$ & $26 \%$ & $1.95 \%$ & $\$$ & $61,042,206$ & $\$ 294,240$ & 8,867 & 9.69 & 59.01 \\
\hline
\end{tabular}

Table 1. Summary statistics for Morgan Stanley trades sorted by Exchange (NYSE and Nasdaq), side of trade where B and S are buy and sell orders respectively, the transaction type where A and V are arrival price and VWAP strategies and $\mathrm{H}, \mathrm{M}$, and $\mathrm{L}$ correspond to high, medium and low urgency trades. Weight denotes the sample fraction of order volume and count is the actual number of orders. Spread denotes the 5-day time weighted bid-ask spread. Volatility is the annualized 21 day volatility. Volume is the size of the order expressed as a fraction of the 21 day median volume. Order value is the dollar size of the order and order shares is the number of shares. Cost is the average transaction cost (TC) expressed in basis points and StDev is the standard deviation of the transaction cost. 


\begin{tabular}{|c|c|c|c|c|c|c|c|c|c|c|c|c|c|c|c|}
\hline Exchange & Benchmark & Urgency & $\begin{array}{l}\text { Volume } \\
\text { Range }\end{array}$ & Weight & Count & Price & Spread & Volatility & Volume & & $\begin{array}{c}\text { pitalization } \\
(000)\end{array}$ & \multicolumn{2}{|c|}{ Order Value } & $\begin{array}{l}\text { Cost } \\
\text { (BP) }\end{array}$ & $\begin{array}{c}\text { StDev } \\
\text { (BP) }\end{array}$ \\
\hline NYSE & A & $\mathrm{H}$ & $\leq 0.25 \%$ & $0.69 \%$ & 2,630 & $\$ 47.77$ & $0.08 \%$ & $22 \%$ & $0.19 \%$ & $\$$ & $94,061,545$ & $\$$ & 190,107 & 4.22 & 10.97 \\
\hline NYSE & A & $M$ & & $3.10 \%$ & 13,664 & $\$ 48.06$ & $0.08 \%$ & $22 \%$ & $0.16 \%$ & $\$$ & $95,714,257$ & $\$$ & 164,767 & 3.69 & 11.64 \\
\hline NYSE & A & L & & $4.17 \%$ & 19,379 & $\$ 50.15$ & $0.08 \%$ & $22 \%$ & $0.14 \%$ & $\$$ & $98,487,506$ & $\$$ & 156,371 & 2.71 & 12.74 \\
\hline NYSE & V & & & $6.54 \%$ & 38,116 & $\$ 47.46$ & $0.08 \%$ & $22 \%$ & $0.13 \%$ & $\$$ & $90,733,082$ & $\$$ & 124,606 & 1.97 & 34.56 \\
\hline NYSE & A & $\mathrm{H}$ & $\leq 0.5 \%$ & $1.69 \%$ & 3,559 & $\$ 51.00$ & $0.08 \%$ & $22 \%$ & $0.37 \%$ & $\$$ & $81,476,811$ & $\$$ & 345,605 & 6.16 & 11.95 \\
\hline NYSE & A & $M$ & & $3.30 \%$ & 9,557 & $\$ 49.09$ & $0.08 \%$ & $23 \%$ & $0.36 \%$ & $\$$ & $68,991,562$ & $\$$ & 250,562 & 5.68 & 15.53 \\
\hline NYSE & A & L & & $2.99 \%$ & 8,027 & $\$ 50.18$ & $0.08 \%$ & $21 \%$ & $0.36 \%$ & $\$$ & $92,105,328$ & $\$$ & 270,537 & 4.15 & 19.65 \\
\hline NYSE & V & & & $5.00 \%$ & 14,890 & $\$ 48.23$ & $0.08 \%$ & $23 \%$ & $0.37 \%$ & $\$$ & $65,205,449$ & $\$$ & 244,088 & 3.06 & 42.27 \\
\hline NYSE & A & $\mathrm{H}$ & $\leq 1.0 \%$ & $2.39 \%$ & 2,979 & $\$ 52.69$ & $0.08 \%$ & $22 \%$ & $0.73 \%$ & $\$$ & $72,154,182$ & $\$$ & 582,738 & 8.93 & 15.98 \\
\hline NYSE & A & $M$ & & $3.64 \%$ & 6,907 & $\$ 48.85$ & $0.08 \%$ & $24 \%$ & $0.72 \%$ & $\$$ & $54,822,342$ & $\$$ & 383,195 & 7.54 & 20.67 \\
\hline NYSE & A & L & & $3.17 \%$ & 6,035 & $\$ 50.22$ & $0.08 \%$ & $22 \%$ & $0.72 \%$ & $\$$ & $82,177,737$ & $\$$ & 381,487 & 6.76 & 28.64 \\
\hline NYSE & V & & & $6.40 \%$ & 11,822 & $\$ 46.88$ & $0.09 \%$ & $23 \%$ & $0.73 \%$ & $\$$ & $71,181,217$ & $\$$ & 393,015 & 5.17 & 47.42 \\
\hline NYSE & A & $\mathrm{H}$ & $>1.0 \%$ & $2.67 \%$ & 2,549 & $\$ 51.53$ & $0.09 \%$ & $23 \%$ & $2.31 \%$ & $\$$ & $48,375,509$ & $\$$ & 760,186 & 14.36 & 25.12 \\
\hline NYSE & A & $M$ & & $7.34 \%$ & 7,052 & $\$ 47.41$ & $0.10 \%$ & $24 \%$ & $3.02 \%$ & $\$$ & $36,941,147$ & $\$$ & 755,825 & 15.55 & 38.97 \\
\hline NYSE & A & L & & $4.77 \%$ & 5,632 & $\$ 47.78$ & $0.10 \%$ & $23 \%$ & $2.55 \%$ & $\$$ & $52,146,046$ & $\$$ & 615,387 & 12.64 & 52.73 \\
\hline NYSE & V & & & $16.88 \%$ & 13,710 & $\$ 45.67$ & $0.09 \%$ & $23 \%$ & $3.95 \%$ & $\$$ & $54,313,103$ & $\$$ & 894,247 & 14.66 & 65.61 \\
\hline NASDAQ & A & $\mathrm{H}$ & $\leq 0.25 \%$ & $0.28 \%$ & 715 & $\$ 36.70$ & $0.06 \%$ & $33 \%$ & $0.18 \%$ & $\$$ & $76,072,230$ & $\$$ & 288,664 & 6.58 & 12.91 \\
\hline NASDAQ & A & $M$ & & $1.59 \%$ & 5,771 & $\$ 37.81$ & $0.06 \%$ & $34 \%$ & $0.15 \%$ & $\$$ & $52,884,846$ & $\$$ & ,367 & 6.05 & 17.13 \\
\hline NASDAQ & A & L & & $1.36 \%$ & 5,065 & $\$ 37.49$ & $0.06 \%$ & $33 \%$ & $0.14 \%$ & $\$$ & $77,185,322$ & $\$$ & 195,338 & 5.29 & 17.93 \\
\hline NASDAQ & V & & & $3.73 \%$ & 16,227 & $\$ 39.02$ & $0.06 \%$ & $34 \%$ & $0.11 \%$ & $\$$ & $71,786,694$ & $\$$ & 167,134 & 3.96 & 42.05 \\
\hline NASDAQ & A & $\mathrm{H}$ & $\leq 0.5 \%$ & $0.82 \%$ & 1,131 & $\$ 39.07$ & $0.06 \%$ & $32 \%$ & $0.37 \%$ & $\$$ & $63,042,552$ & $\$$ & 527,242 & 10.76 & 18.02 \\
\hline NASDAQ & $A$ & $M$ & & $1.35 \%$ & 4,455 & $\$ 36.72$ & $0.08 \%$ & $36 \%$ & $0.36 \%$ & $\$$ & $30,091,409$ & $\$$ & 220,816 & 9.15 & 21.53 \\
\hline NASDAQ & A & $\mathrm{L}$ & & $0.89 \%$ & 2,578 & $\$ 38.02$ & $0.07 \%$ & $36 \%$ & $0.37 \%$ & $\$$ & $37,310,972$ & $\$$ & 251,330 & 8.33 & 31.04 \\
\hline NASDAQ & V & & & $1.64 \%$ & 5,597 & $\$ 35.82$ & $0.07 \%$ & $36 \%$ & $0.36 \%$ & $\$$ & $45,881,902$ & $\$$ & 213,288 & 6.07 & 59.83 \\
\hline NASDAQ & A & $\mathrm{H}$ & $\leq 1.0 \%$ & $0.85 \%$ & 992 & $\$ 38.30$ & $0.07 \%$ & $35 \%$ & $0.70 \%$ & $\$$ & $42,108,883$ & $\$$ & 623,028 & 15.81 & 21.17 \\
\hline NASDAQ & A & $M$ & & $1.31 \%$ & 3,453 & $\$ 36.58$ & $0.09 \%$ & $38 \%$ & $0.71 \%$ & $\$$ & $15,709,279$ & $\$$ & 275,812 & 14.78 & 30.01 \\
\hline NASDAQ & A & $\mathrm{L}$ & & $0.97 \%$ & 2,003 & $\$ 39.11$ & $0.07 \%$ & $37 \%$ & $0.72 \%$ & $\$$ & $32,386,856$ & $\$$ & 352,916 & 12.36 & 45.50 \\
\hline NASDAQ & V & & & $1.80 \%$ & 5,310 & $\$ 34.08$ & $0.08 \%$ & $36 \%$ & $0.73 \%$ & $\$$ & $36,671,191$ & $\$$ & 246,208 & 10.62 & 66.66 \\
\hline NASDAQ & $A$ & $\mathrm{H}$ & $>1.0 \%$ & $0.83 \%$ & 1,061 & $\$ 37.41$ & $0.10 \%$ & $36 \%$ & $2.91 \%$ & $\$$ & $10,249,188$ & $\$$ & 565,871 & 26.99 & 47.16 \\
\hline NASDAQ & A & $M$ & & $2.26 \%$ & 3,236 & $\$ 32.85$ & $0.11 \%$ & $39 \%$ & $3.10 \%$ & $\$$ & $8,045,030$ & $\$$ & 508,137 & 22.96 & 58.51 \\
\hline NASDAQ & A & L & & $1.91 \%$ & 2,869 & $\$ 36.91$ & $0.10 \%$ & $38 \%$ & $2.85 \%$ & $\$$ & $15,236,379$ & $\$$ & 484,223 & 26.02 & 79.01 \\
\hline NASDAQ & V & & & $3.62 \%$ & 6,942 & $\$ 33.30$ & $0.11 \%$ & $37 \%$ & $3.46 \%$ & $\$$ & $23,073,584$ & $\$$ & 379,150 & 24.65 & 94.67 \\
\hline
\end{tabular}

Table 2. Summary statistics for Morgan Stanley trades sorted by Exchange (NYSE and Nasdaq), side of trade where B and S are buy and sell orders respectively, the transaction type where A and V are arrival price and VWAP strategies and $\mathrm{H}, \mathrm{M}$, and L correspond to high, medium and low urgency trades. Weight denotes the sample fraction of order volume and count is the actual number of orders. Spread denotes the 5-day time weighted bid-ask spread. Volatility is the annualized 21 day volatility. Volume is the size of the order expressed as a fraction of the 21 day median volume. Order value is the dollar size of the order and order shares is the number of shares. Cost is the average transaction cost (TC) expressed in basis points and StDev is the standard deviation of the transaction cost. 


\begin{tabular}{|lll|}
\hline & & \\
VARIABLE & COEFFICIENT & ROBUST T-STAT \\
Const & 11.80559 & 90.86393 \\
Spread & 1.815802 & 14.39896 \\
Log volatility & 1.207152 & 64.98954 \\
Log volume & -0.51614 & -55.6044 \\
Log value & 0.536306 & 46.08766 \\
Low urg & -1.45436 & -83.2275 \\
Med urg & -1.92541 & -61.058 \\
High urg & -2.33731 & -88.2665 \\
\hline
\end{tabular}

Table 3. Variance parameter estimates for NYSE stocks. The spread is the time weighted 5 day bid ask spread, volatility is the annualized lagged 21 day close to close volatility, volume is the 21 day median daily volume and value is the dollar size of the order. Low, medium, and high urgency are dummy variables for the corresponding trade type.

\begin{tabular}{|lll|}
\hline & & \\
VARIABLE & COEFFICIENT & ROBUST T-STAT \\
Const & 5.173827 & 30.0342 \\
Spread & 0.969804 & 8.395586 \\
Log volatility & 0.503987 & 21.14475 \\
Log volume & -0.47084 & -43.4163 \\
Log value & 0.43783 & 40.27979 \\
Low urg & 0.094929 & 2.41284 \\
Med urg & 0.305623 & 8.796438 \\
High urg & 0.41034 & 11.28093 \\
\hline
\end{tabular}

Table 4. Mean parameter estimates for NYSE stocks. The spread is the time weighted 5 day bid ask spread, volatility is the annualized lagged 21 day close to close volatility, volume is the 21 day median daily volume and value is the dollar size of the order. Low, medium, and high urgency are dummy variables for the corresponding trade type. 


\begin{tabular}{|lll|}
\hline & & \\
VARIABLE & COEFFICIENT & ROBUST T-STAT \\
Const & 11.40519 & 71.44173 \\
Spread & 2.016026 & 17.00666 \\
Log volatility & 1.078963 & 40.27656 \\
Log volume & -0.44182 & -46.7497 \\
Log value & 0.453704 & 42.64865 \\
Low urg & -1.04398 & -42.1013 \\
Med urg & -1.70511 & -65.0131 \\
High urg & -2.10623 & -48.7398 \\
\hline
\end{tabular}

Table 5. Variance parameter estimates for Nasdaq stocks. The spread is the time weighted 5 day bid ask spread, volatility is the annualized lagged 21 day close to close volatility, volume is the 21 day median daily volume and value is the dollar size of the order. Low, medium, and high urgency are dummy variables for the corresponding trade type.

\begin{tabular}{|lll|}
\hline & & \\
VARIABLE & COEFFICIENT & ROBUST T-STAT \\
Const & 5.354067 & 26.04098 \\
Spread & 1.014023 & 8.734035 \\
Log volatility & 0.513628 & 16.96502 \\
Log volume & -0.41447 & -29.9304 \\
Log value & 0.376208 & 24.99588 \\
Low urg & 0.025356 & 0.243943 \\
Med urg & 0.230764 & 5.716912 \\
High urg & 0.282479 & 6.156517 \\
\hline
\end{tabular}

Table 6. Mean parameter estimates for Nasdaq stocks. The spread is the time weighted 5 day bid ask spread, volatility is the annualized lagged 21 day close to close volatility, volume is the 21 day median daily volume and value is the dollar size of the order. Low, medium, and high urgency are dummy variables for the corresponding trade type. 


\begin{tabular}{|c|c|c|}
\hline & Variance & \\
\hline VARIABLE & NYSE & Nasdaq \\
\hline Const & $\begin{array}{l}12.31 \\
(156.06)\end{array}$ & $\begin{array}{l}12.53 \\
(140.53)\end{array}$ \\
\hline Spread & $\begin{array}{l}1.201 \\
(17.82)\end{array}$ & $\begin{array}{l}0.816 \\
(11.22)\end{array}$ \\
\hline Log volatility & $\begin{array}{l}1.60 \\
(95.73)\end{array}$ & $\begin{array}{l}1.617 \\
(62.47)\end{array}$ \\
\hline Log volume & $\begin{array}{l}-0.811 \\
(-237.12)\end{array}$ & $\begin{array}{l}-0.681 \\
(-165.61)\end{array}$ \\
\hline Log value & $\begin{array}{l}0.737 \\
(33.51)\end{array}$ & $\begin{array}{l}0.639 \\
(132.96)\end{array}$ \\
\hline Low urg & $\begin{array}{l}1.924 \\
(32.44)\end{array}$ & $\begin{array}{l}1.22 \\
(15.64)\end{array}$ \\
\hline Med urg & $\begin{array}{l}1.444 \\
(24.67)\end{array}$ & $\begin{array}{l}0.652 \\
(8.28)\end{array}$ \\
\hline High urg & $\begin{array}{l}0.998 \\
(16.66)\end{array}$ & $\begin{array}{l}0.146 \\
(1.81)\end{array}$ \\
\hline Value & $\begin{array}{l}3.58 * 10^{-8} \\
(6.16)\end{array}$ & $\begin{array}{l}5.89 * 10^{-8} \\
(6.47)\end{array}$ \\
\hline Volatility & $\begin{array}{l}-1.572 \\
(-25.56)\end{array}$ & $\begin{array}{l}-0.412 \\
(-2.04)\end{array}$ \\
\hline Log Spread & $\begin{array}{l}0.070 \\
(8.31)\end{array}$ & $\begin{array}{l}0.182 \\
(15.22)\end{array}$ \\
\hline Volume & $\begin{array}{l}7.55 * 10^{-10} \\
(27.47)\end{array}$ & $\begin{array}{l}2.03 * 10^{-10} \\
(16.98)\end{array}$ \\
\hline VWAP*Log Volatility & $\begin{array}{l}0.0367 \\
(3.85)\end{array}$ & $\begin{array}{l}-0.12 \\
(-8.81)\end{array}$ \\
\hline VWAP*Log Volume & $\begin{array}{l}0.462 \\
(135.68)\end{array}$ & $\begin{array}{l}.3624 \\
(89.54)\end{array}$ \\
\hline VWAP*Log Value & $\begin{array}{l}-0.398 \\
(112.21)\end{array}$ & $\begin{array}{l}-0.350 \\
(-60.99)\end{array}$ \\
\hline
\end{tabular}

Table 7. Variance model estimates for NYSE and Nasdaq expanded models. Numbers in parenthesis are t-stats. The spread is the time weighted 5 day bid ask spread, volatility is the annualized lagged 21 day close to close volatility, volume is the 21 day median daily volume and value is the dollar size of the order. Low, medium, and high urgency are dummy variables for the corresponding trade type 


\begin{tabular}{|c|c|c|}
\hline \multicolumn{3}{|c|}{ Expected Cost } \\
\hline VARIABLE & NYSE & Nasdaq \\
\hline Const & $\begin{array}{l}6.61 \\
(16.04)\end{array}$ & $\begin{array}{l}5.69 \\
(20.36)\end{array}$ \\
\hline Spread & $\begin{array}{l}0.817 \\
(6.10)\end{array}$ & $\begin{array}{l}0.673 \\
(3.81)\end{array}$ \\
\hline Log volatility & $\begin{array}{l}0.525 \\
(22.23)\end{array}$ & $\begin{array}{l}0.661 \\
(7.47)\end{array}$ \\
\hline Log volume & $\begin{array}{l}-0.4466 \\
(-43.76)\end{array}$ & $\begin{array}{l}-0.383 \\
(-28.5)\end{array}$ \\
\hline Log value & $\begin{array}{l}0.406 \\
(33.51)\end{array}$ & $\begin{array}{l}0.344 \\
(24.01)\end{array}$ \\
\hline Low urg & $\begin{array}{l}-0.995 \\
(-2.44)\end{array}$ & $\begin{array}{l}0.199 \\
(2.21)\end{array}$ \\
\hline Med urg & $\begin{array}{l}-0.799 \\
(-1.96)\end{array}$ & $\begin{array}{l}0.418 \\
(4.87)\end{array}$ \\
\hline High urg & $\begin{array}{l}-0.699 \\
(-1.71)\end{array}$ & $\begin{array}{l}0.475 \\
(5.40)\end{array}$ \\
\hline Value & $\begin{array}{l}5.31 * 10^{-8} \\
(3.90)\end{array}$ & \\
\hline Volatility & & $\begin{array}{l}-0.412 \\
(-2.04)\end{array}$ \\
\hline Log Spread & & $\begin{array}{l}8.62 * 10^{-8} \\
(4.81)\end{array}$ \\
\hline VWAP*Log Volatility & $\begin{array}{l}0.0481 \\
(1.69)\end{array}$ & \\
\hline VWAP*Log Volume & $\begin{array}{l}-0.0991 \\
(-3.72)\end{array}$ & $\begin{array}{l}-0.2236 \\
(-2.96)\end{array}$ \\
\hline
\end{tabular}

Table 8. Expected cost model estimates for NYSE and Nasdaq expanded models. Numbers in parenthesis are t-stats. The spread is the time weighted 5 day bid ask spread, volatility is the annualized lagged 21 day close to close volatility, volume is the 21 day median daily volume and value is the dollar size of the order. Low, medium, and high urgency are dummy variables for the corresponding trade type. 


\begin{tabular}{|lll|ll|ll|}
\hline & TYPICAL & & \multicolumn{2}{c|}{ 10th Percentile Value } & \multicolumn{2}{c|}{ 90th Percentile Value } \\
& NYSE & Nasdaq & NYSE & Nasdaq & NYSE & Nasdaq \\
\hline VWAP & $0-3.7$ & $0-1.5$ & $0-3.0$ & $0-1.1$ & $0-5.8$ & $0-2.4$ \\
Low & $3.7-65.8$ & $1.5-54.4$ & $3.0-98.2$ & $1.1-83.2$ & $5.8-41.4$ & $2.4-33.8$ \\
Med & $65.8-71.1$ & & $98.2-106.3$ & & $41.4-44.8$ & \\
High & $>71.1$ & $>54.4$ & $>106.3$ & $>83.2$ & $>44.8$ & $>33.8$ \\
\hline
\end{tabular}

Table 9. This table presents the risk aversion values that are consistent with a given trading strategy optimizing the quadratic utility under average conditions for average volume (left) $10^{\text {th }}$ percentile Value, or large trades, (middle) and $90^{\text {th }}$ percentile, or small trades, (right). 




Figure 1: NYSE average cost/risk tradeoff for different order sizes $(<.25 \%, .25-.5 \%$, .5-1\%, and $>1 \%$ ). The order size is expressed as a fraction of the median 21 day daily volume. Each contour has four points - high urgency, medium urgency, low urgency, and VWAP (moving from left to right).

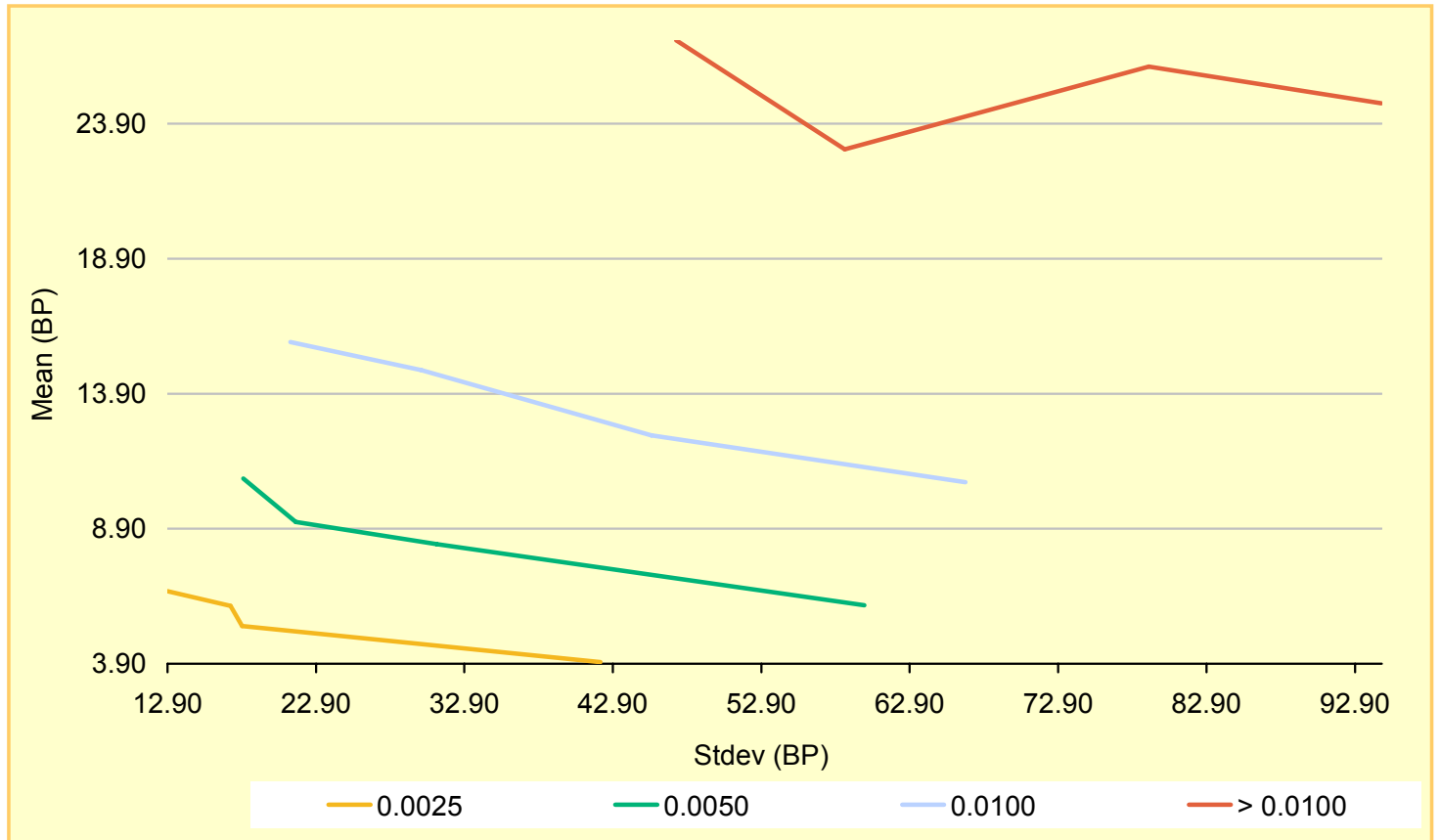

Figure 2: Nasdaq average cost/risk tradeoff for different order sizes $(<.25 \%, .25-.5 \%, .5-1 \%$, and $>1 \%$ ). The order size is expressed as a fraction of the median 21 day daily volume. Each contour has four points - high urgency, medium urgency, low urgency, and VWAP (moving from left to right). 




Figure 3: Expected cost as a function of the order size expressed as a fraction of average daily volume for an NYSE stock under "normal” conditions.

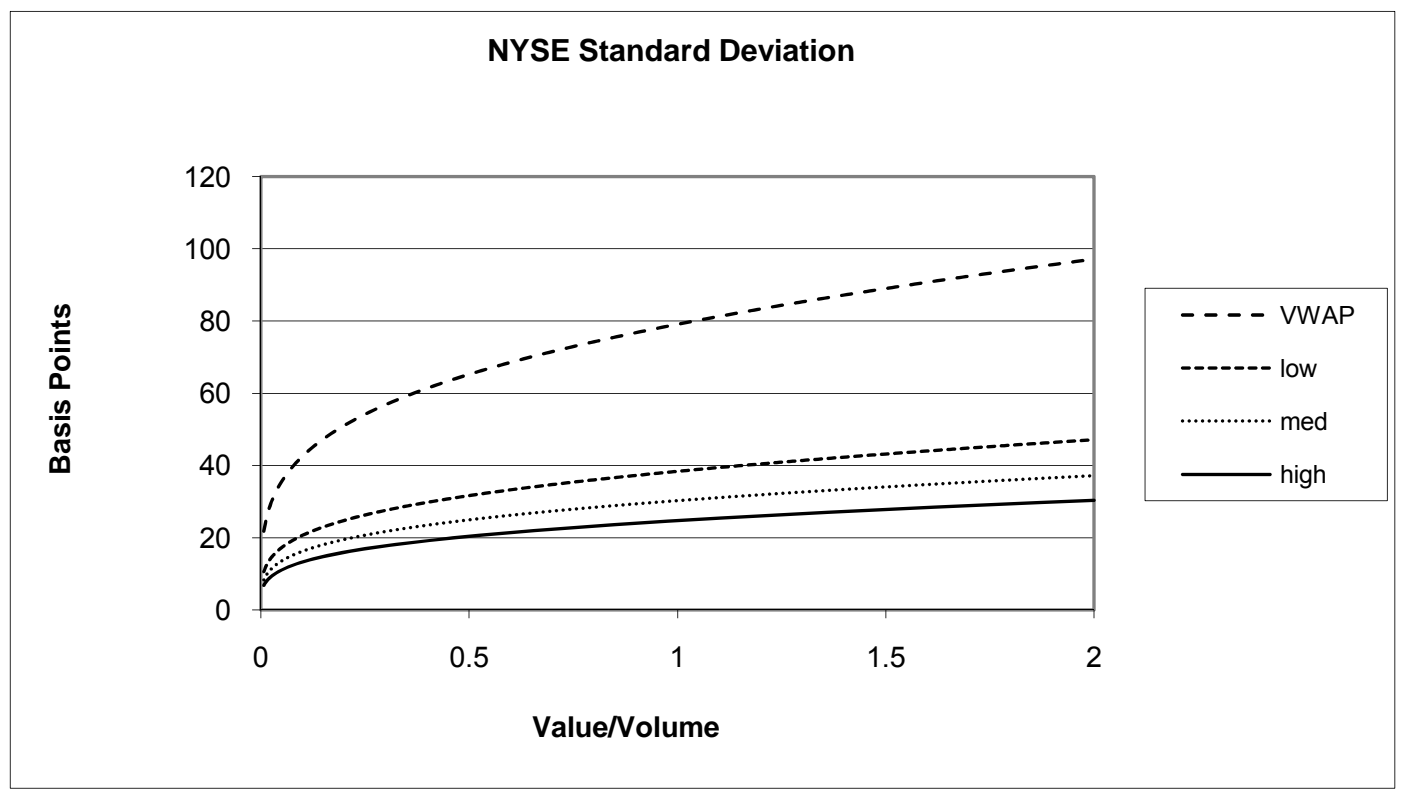

Figure 4: Standard deviation of transaction cost as a function of the order size expressed as a fraction of average daily volume for an NYSE stock under "normal” conditions. 


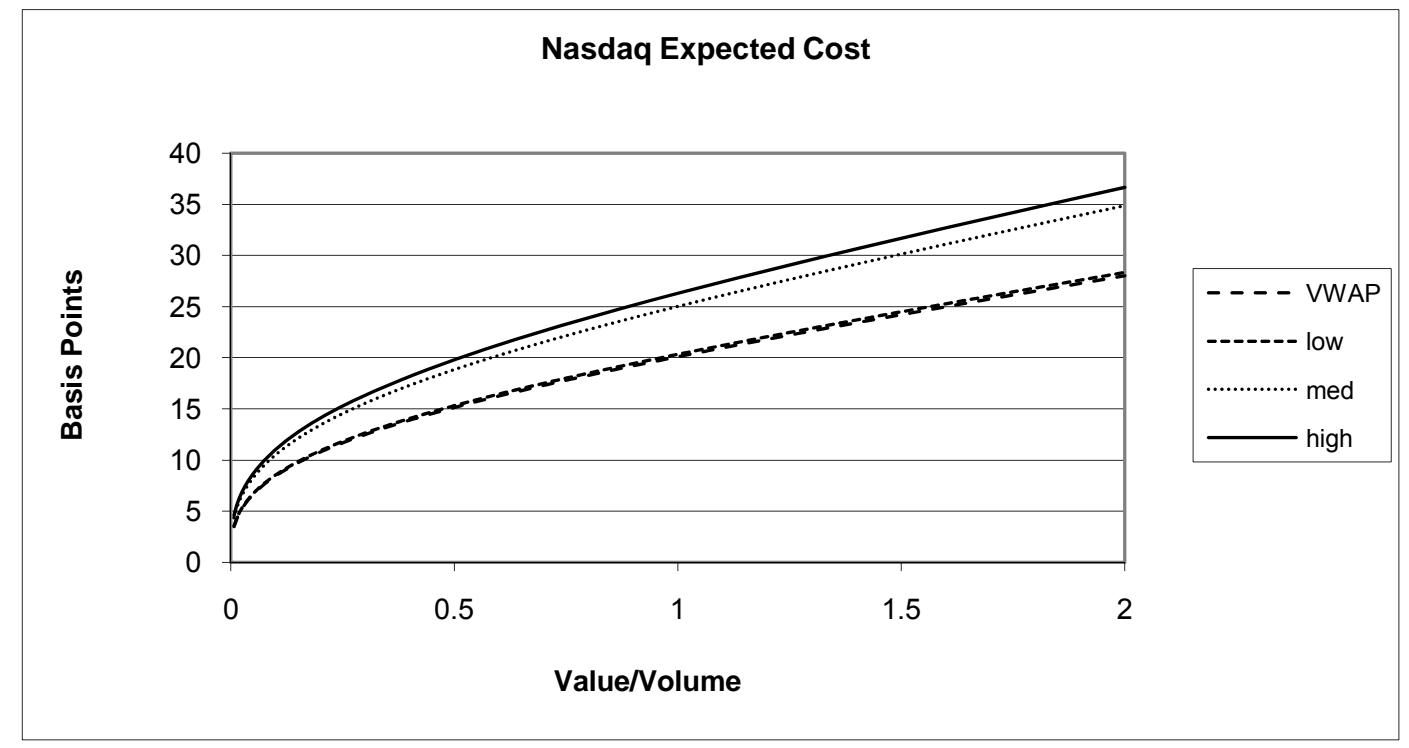

Figure 5. Expected Cost as a function of the order size expressed as a fraction of average daily volume for a Nasdaq stock under "normal” conditions.

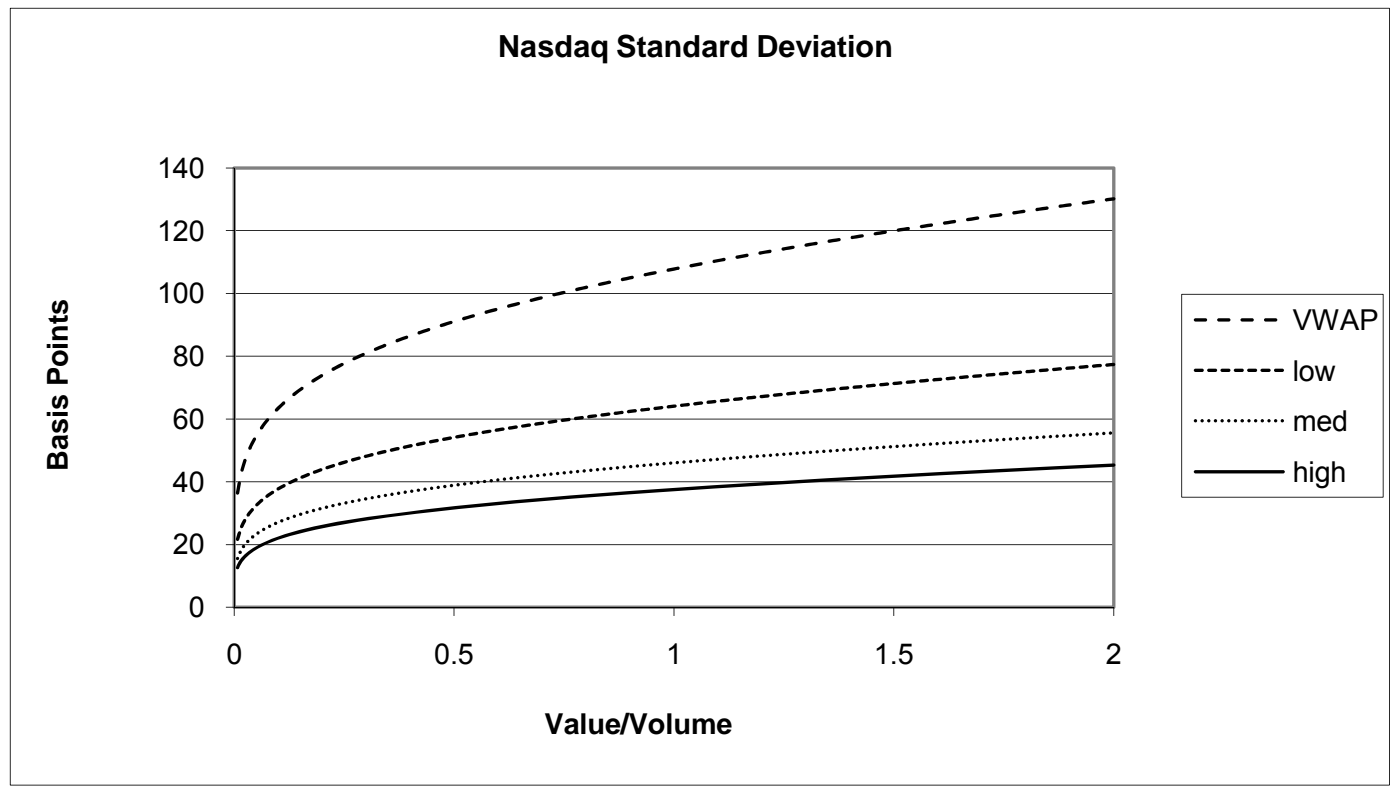

Figure 6. Standard deviation of transaction cost as a function of the order size expressed as a fraction of average daily volume for a Nasdaq stock under "normal" conditions. 


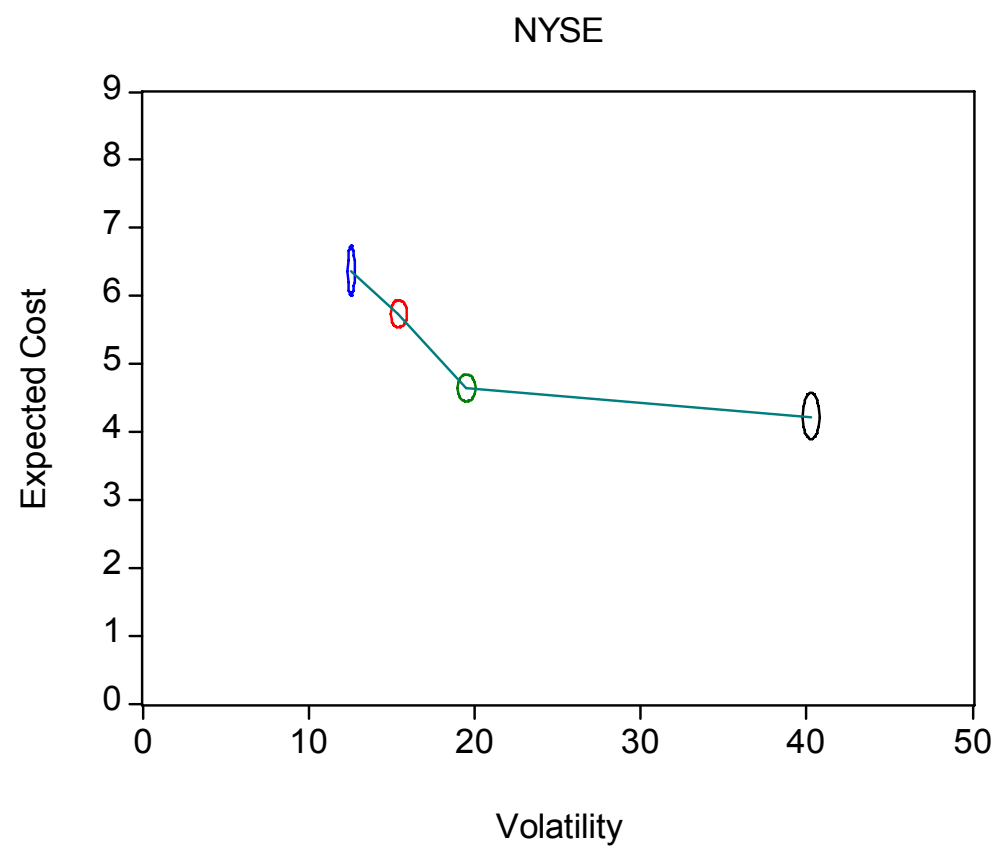

Figure 7. Expected cost and risk frontier for a typical NYSE stock on a typical day. Ellipses denote 95\% confidence intervals around high urgency, medium urgency, low urgency, and VWAP order types (from left to right) under “normal” conditions.

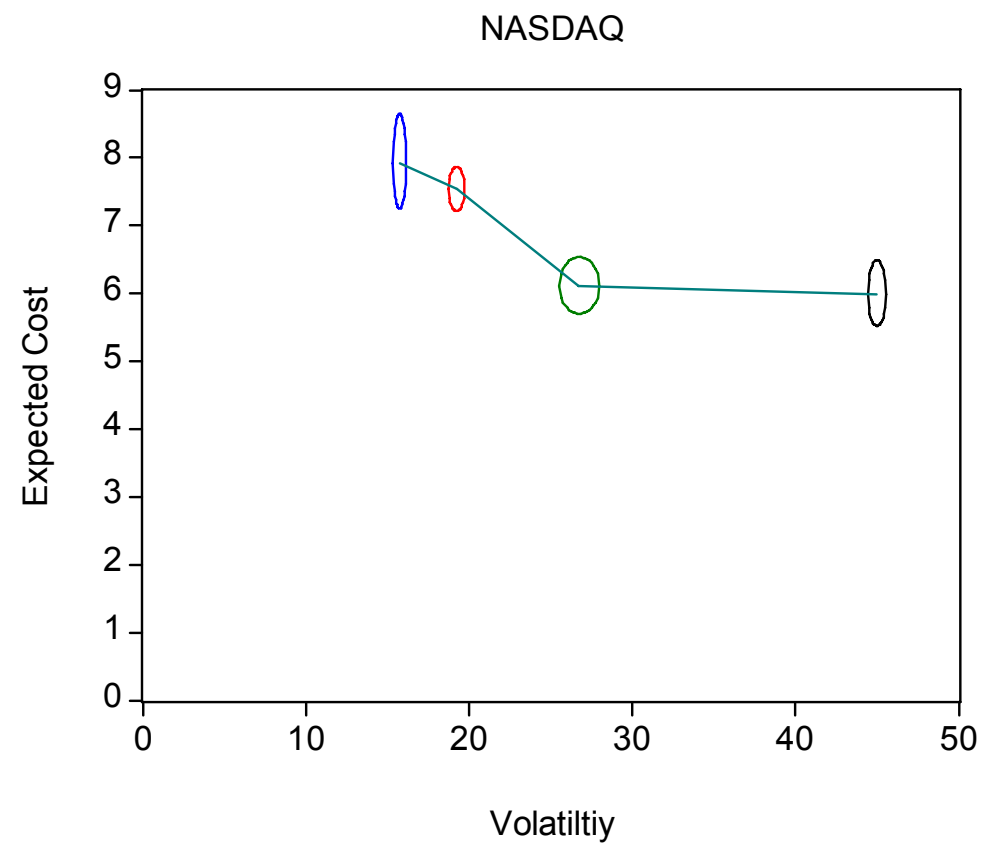

Figure 8. Expected cost and risk frontier for a typical Nasdaq stock on a typical day. Ellipses denote 95\% confidence intervals around high urgency, medium urgency, low urgency, and VWAP order types (from left to right) under “normal” conditions. 


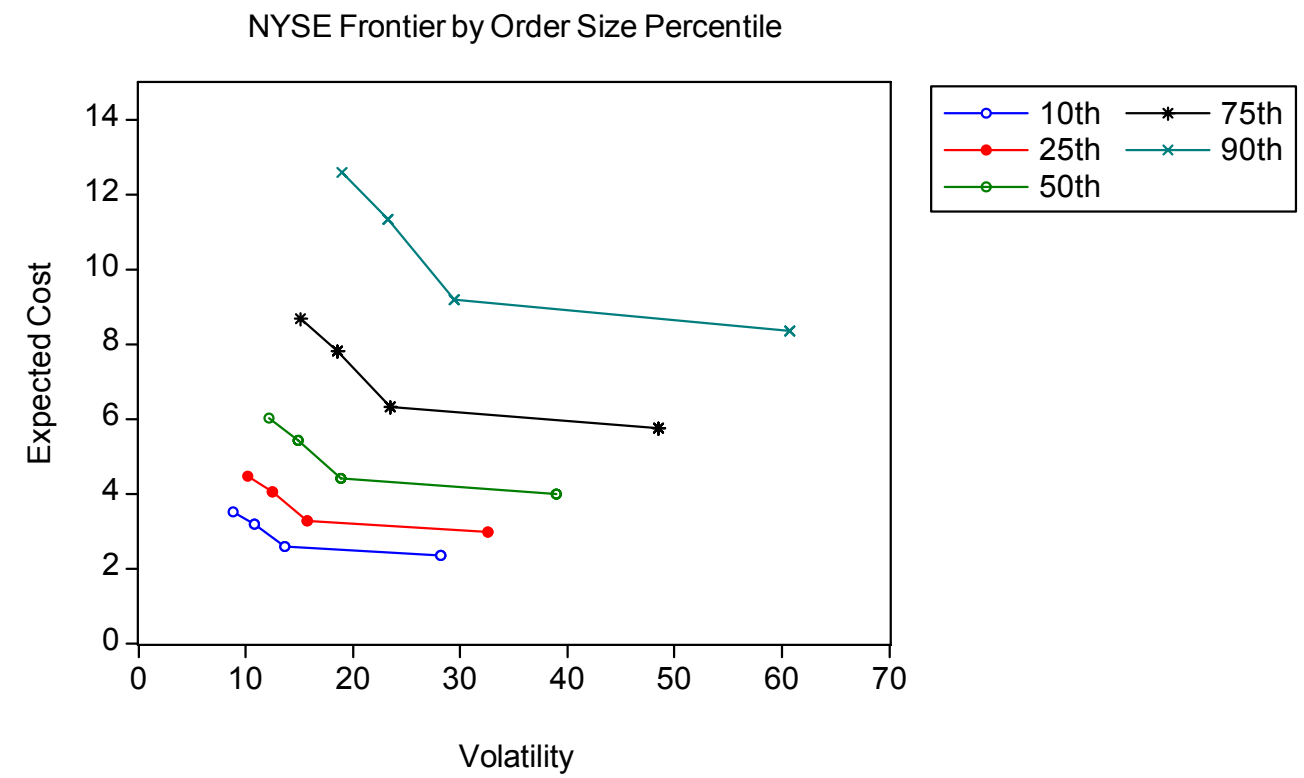

Figure 9. Expected cost/risk frontier for a typical NYSE stock on a typical day. Each contour has four points corresponding to high urgency, medium urgency, low urgency, and VWAP orders (from left to right) representing the frontier for different quantiles of order size expressed as a fraction of average daily volume.

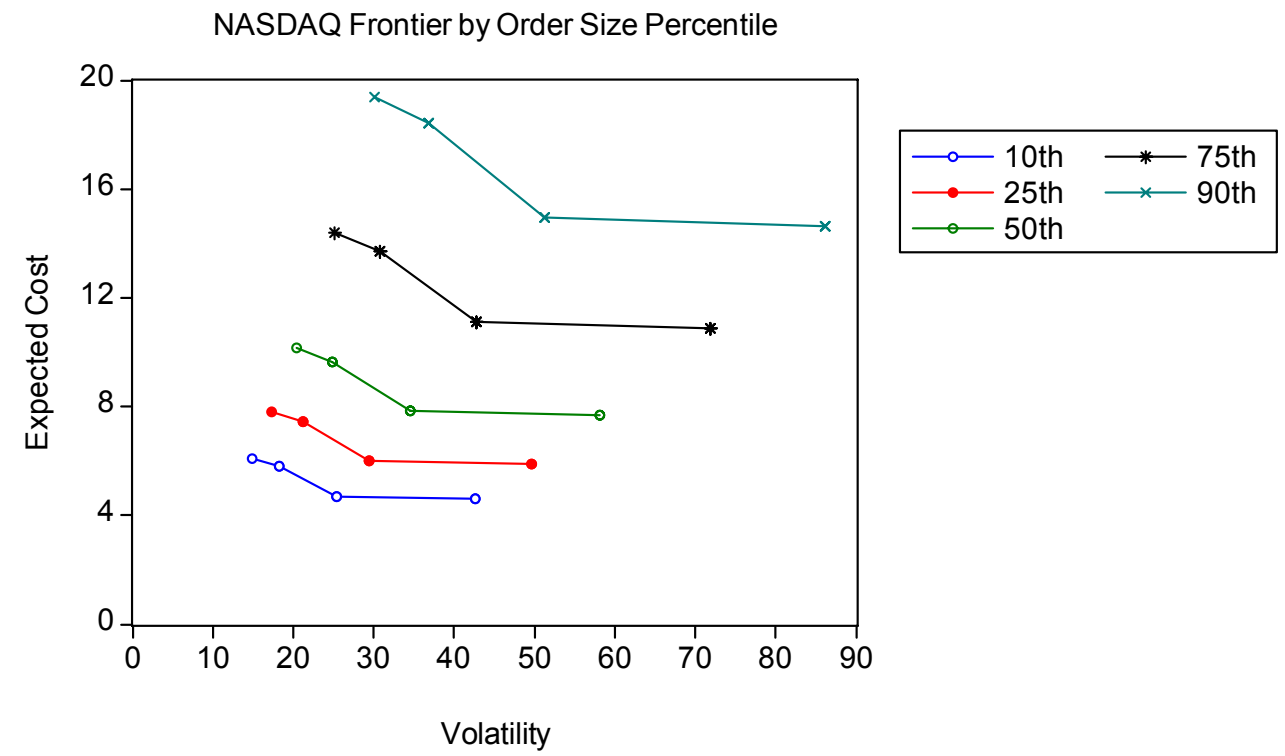

Figure 10. Expected cost/risk frontier for a typical Nasdaq stock on a typical day. Each contour has four points corresponding to high urgency, medium urgency, low urgency, and VWAP orders (from left to right) representing the frontier for different quantiles of order size expressed as a fraction of average daily volume. 


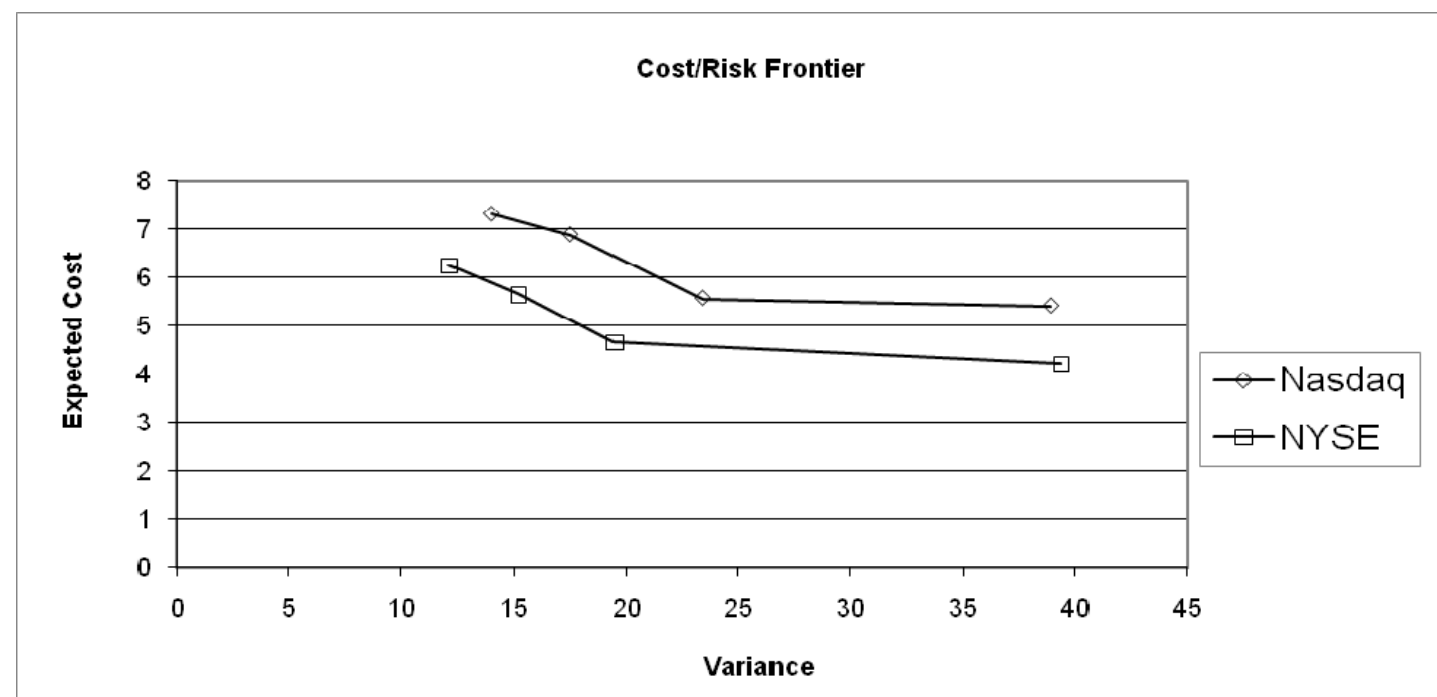

Figure 11. Nasdaq and NYSE mean variance transaction cost frontiers for identical trades under identical conditions. Each contour has four points corresponding to high urgency, medium urgency, low urgency, and VWAP orders (from left to right).

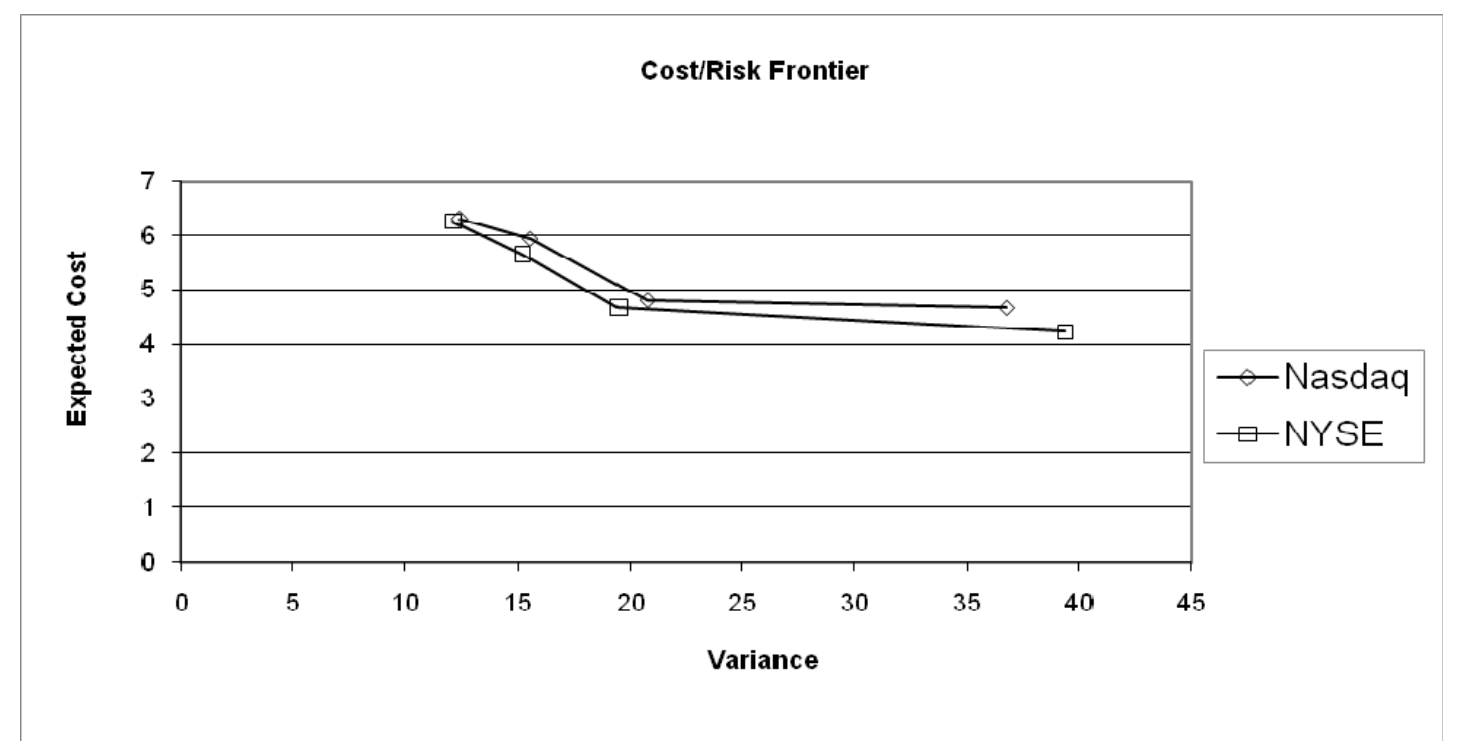

Figure 12. Nasdaq and NYSE mean variance transaction cost frontiers for identical trades under identical conditions assuming that Nasdaq volume is 30\% overstated. Each contour has four points corresponding to high urgency, medium urgency, low urgency, and VWAP orders (from left to right). 


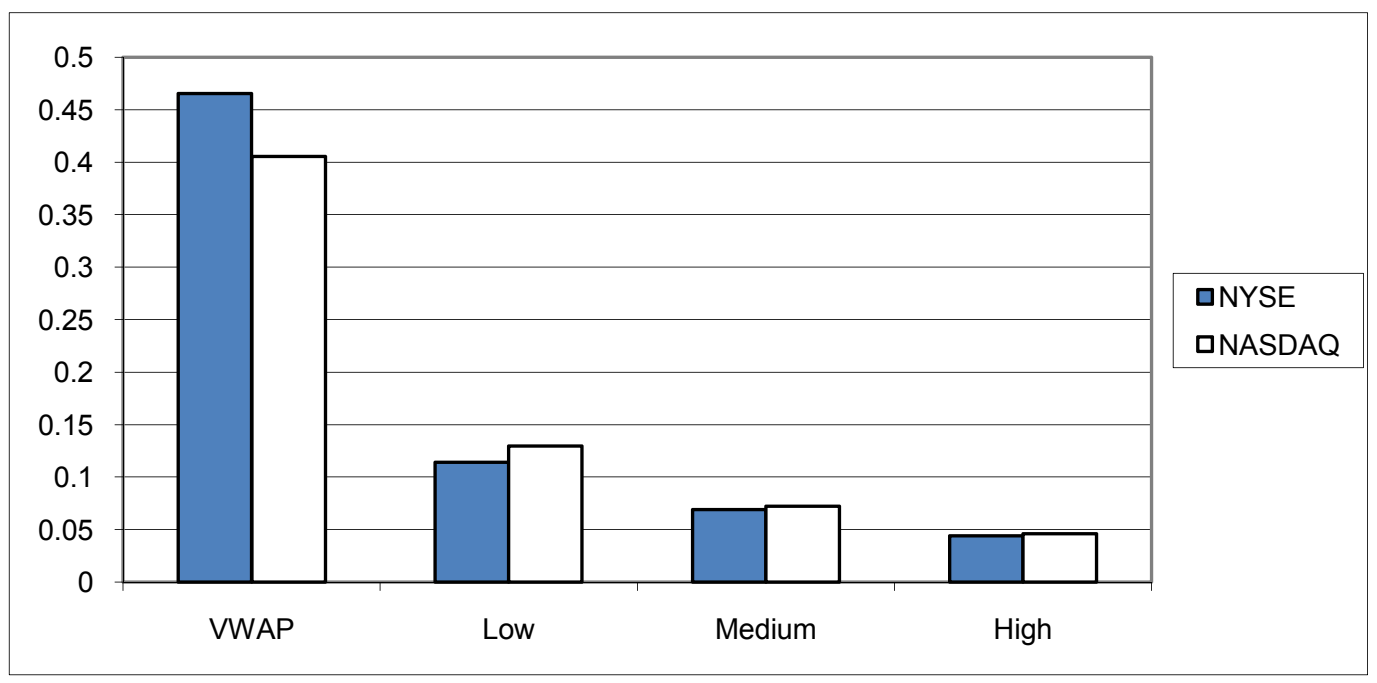

Figure 13. The cost of risk for the VWAP, Low, Medium, and High urgency orders for a quadratic utility investor with risk aversion parameter of 3 for NYSE and Nasdaq stocks.

NYSE

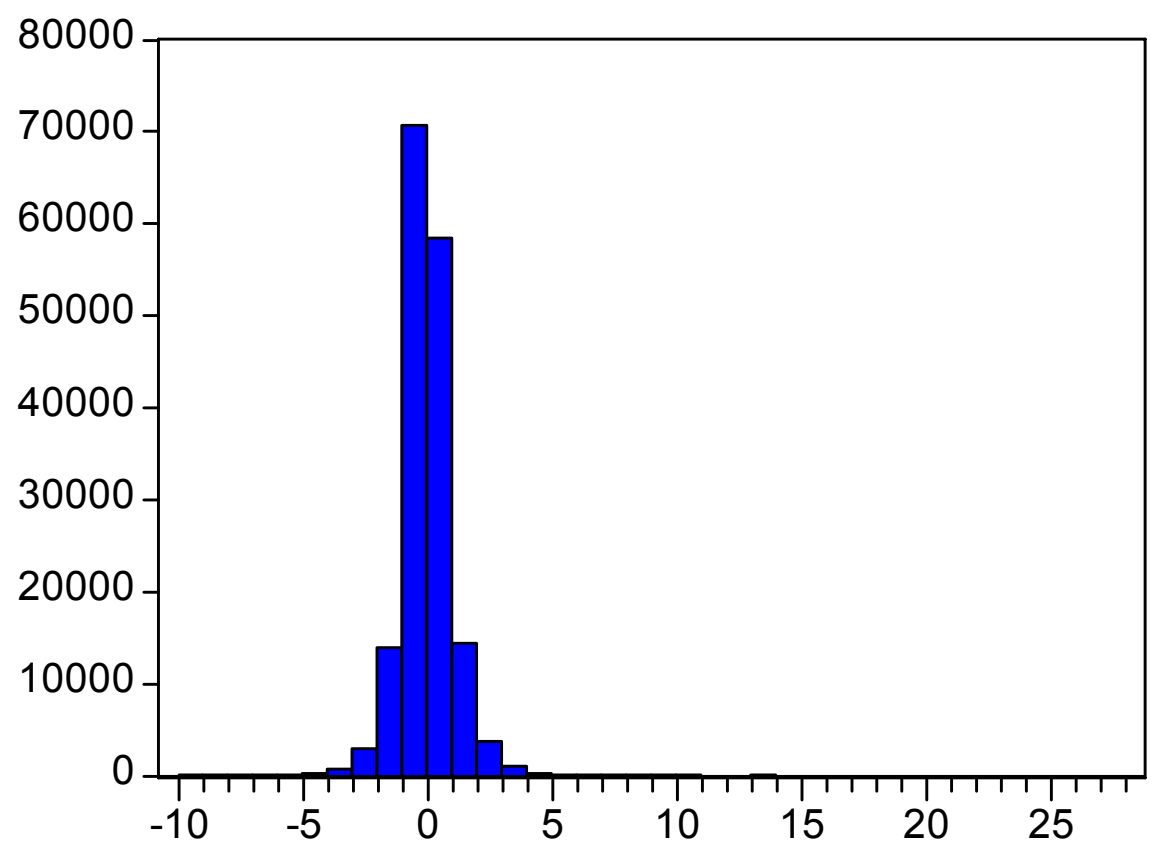

Figure 14. Standardized residuals from the cost model for NYSE stocks. 


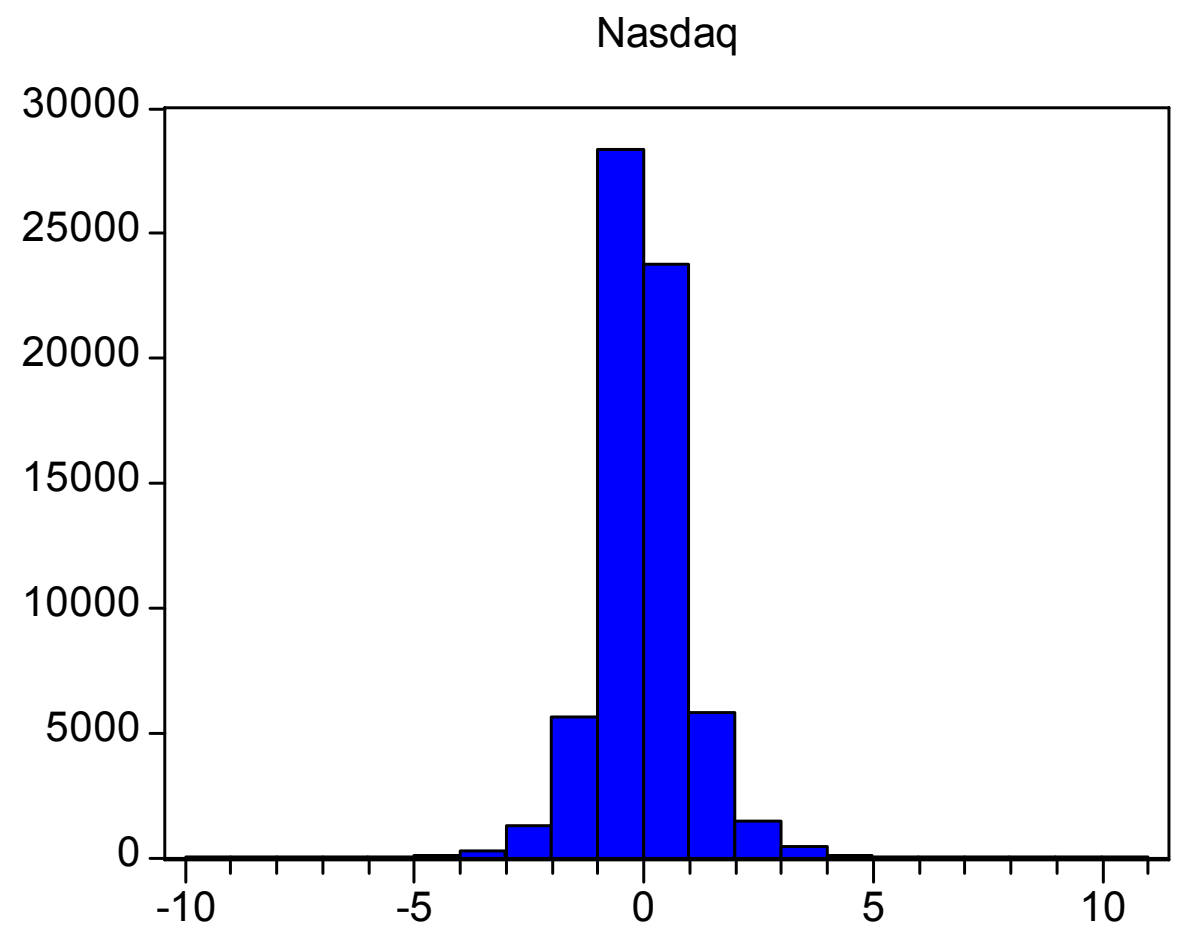

Figure 15. Standardized residuals from the cost model for Nasdaq stocks. 


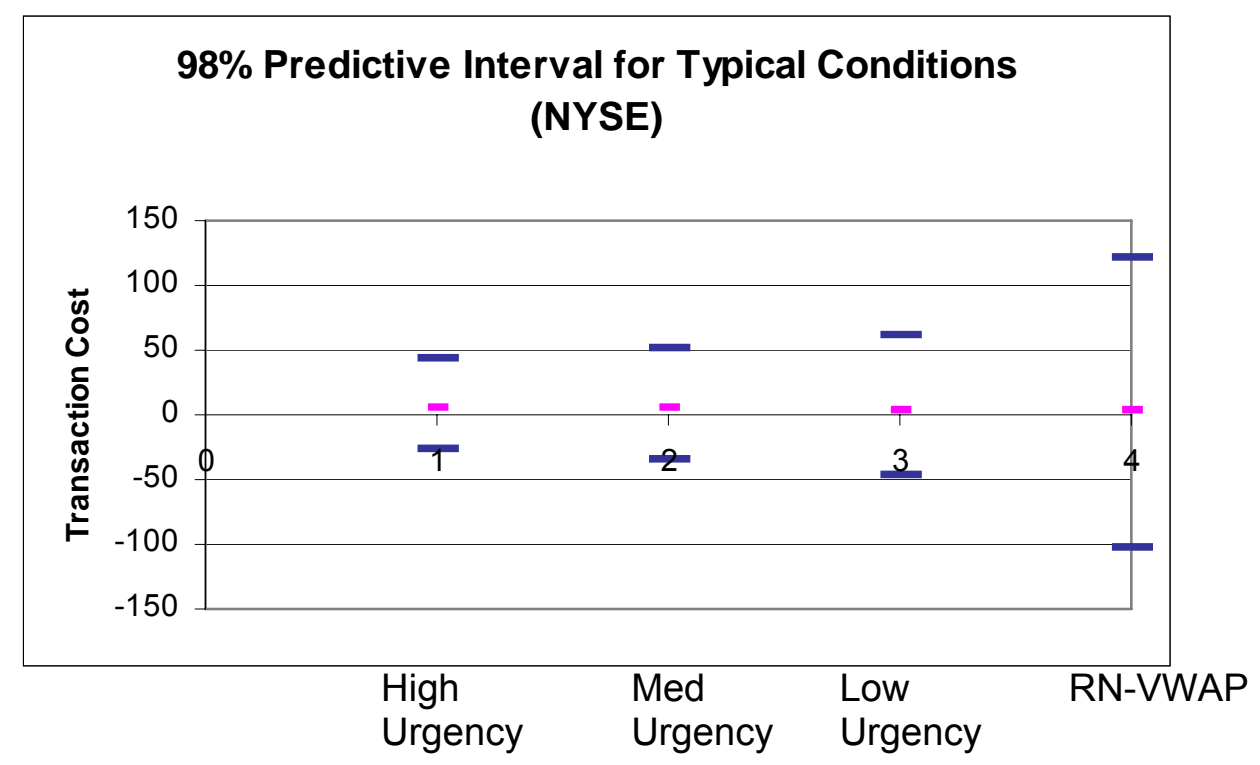

IFigure 16. This plot shows the 98\% predictive interval for the transaction cost for a typical NYSE stock on a typical day. 0 corresponds to VWAP, 1 to low urgency, 2 to medium urgency and 3 to high urgency. For each trade type, the upper bar denotes the $1 \%$ LVAR.

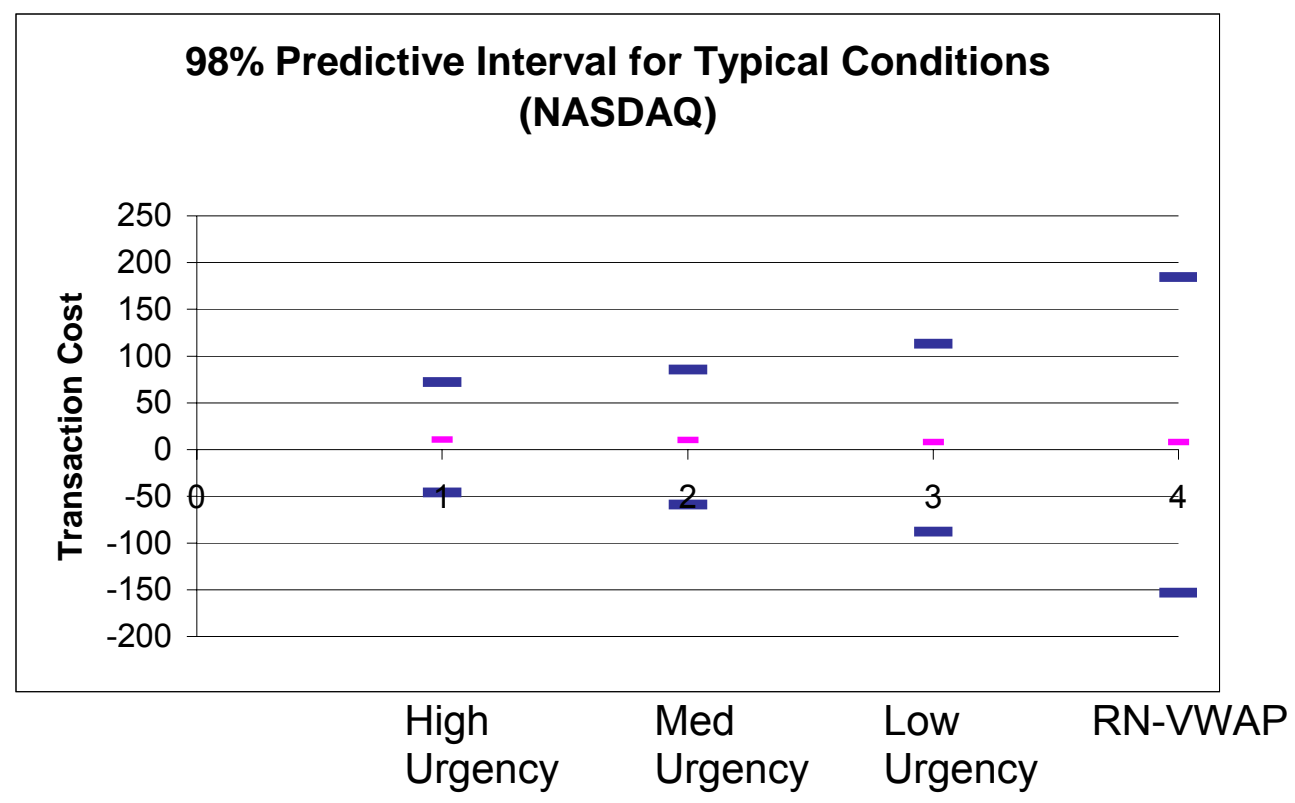

Figure 17. This plot shows the 98\% predictive interval for the transaction cost for a typical Nasdaq stock on a typical day. 0 corresponds to VWAP, 1 to low urgency, 2 to medium urgency and 3 to high urgency. For each trade type, the upper bar denotes the 1\% LVAR. 
References

Acharya, Viral and Lasse Pederson(2005), “Asset Pricing with Liquidity Risk” Journal of Financial Economics,vol 11, pp.375-410

Almgren, Robert and Neil Chriss, (1999) “Value under Liquidation”, Risk, 12

Almgren, Robert and Neil Chriss,(2000) “Optimal Execution of Portfolio Transactions,” Journal of Risk, 3, pp 5-39

Almgren, Robert,(2003) "Optimal Execution with Nonlinear Impact Functions and Tradingenhanced Risk”, Applied Mathematical Finance, 10,pp1-18

Bertsimas, Dimitris, and Andrew W. Lo, (1998) “Optimal Control of Execution Costs,” Journal of Financial Markets, 1, pp1-50

Bessembinder, Hendrik, (2003) "Issues in Assessing Trade Execution Costs”, Journal of Financial Markets, Vol 6, pp 233-257

Chan, Louis K. and Josef Lakonishok, (1995) “The Behavior of Stock Prices around Institutional Trades”, Journal of Finance, Vol 50, No. 4, pp1147-1174

Chou, Ray, Robert Engle and Alex Kane,(1992)”Measuring risk aversion from excess returns on a stock index", Journal of Econometrics, 52, pp201-224

Easley, David, Soeren Hvidkjaer, and Maureen O’Hara, 2002, Is information risk a determinant of asset returns? Journal of Finance 57, 2185-2222.

Engle, Robert, and Robert Ferstenberg, 2007, Execution Risk, J ournal of Portfolio Management, V33, 12, pp 34-45

Friend, Irwin and Marshall Blume,(1975) “The Demand for Risky Assets”, American Economic Review, Vol65, pp 900-922

Grinold R. and R. Kahn(1999) Active Portfolio Management ( $2^{\text {nd }}$ Edition) Chapter 16 pp473-475, McGraw-Hill

Lehmann, Bruce,(2003) “What We Measure in Execution Cost Measurement”, Journal of Financial Markets, Vol 6, pp 227-231

Obizhaeva, Anna and Jiang Wang (2005) “Optimal Trading Strategy and Supply/Demand Dynamics” manuscript

Pastor, Lubos, Robert Stambaugh, (2003), “Liquidity Risk and Expected Stock Returns”, Journal of Political Economy 111, 642-685.

Peterson, Mark and Eric Sirri, (2003) "Evaluation of the Biases in Execution Cost Estimation Using Trade and Quote Data”, Journal of Financial Markets, Vol 6, pp 259-280 
Werner, Ingrid, (2003) “NYSE Order Flow, Spreads and Information”, Journal of Financial Markets, Vol 6, pp 309-335

White, Halbert, 1982, Maximum Likelihood Estimation of Misspecified Models, Econometrica, 50, 1-25. 